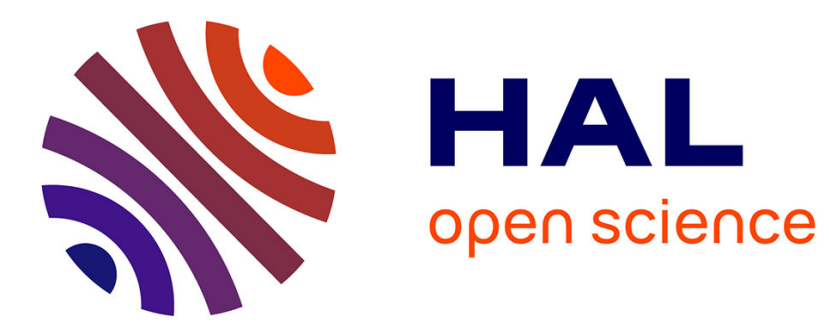

\title{
Turbulence topology evolution in weakly turbulent premixed flames
}

Arnaud Mura, Song Zhao

\section{To cite this version:}

Arnaud Mura, Song Zhao. Turbulence topology evolution in weakly turbulent premixed flames. Physics of Fluids, 2021, 33 (3), pp.035110. 10.1063/5.0039330 . hal-03442314v2

\section{HAL Id: hal-03442314 \\ https://hal.science/hal-03442314v2}

Submitted on 23 Nov 2021

HAL is a multi-disciplinary open access archive for the deposit and dissemination of scientific research documents, whether they are published or not. The documents may come from teaching and research institutions in France or abroad, or from public or private research centers.
L'archive ouverte pluridisciplinaire HAL, est destinée au dépôt et à la diffusion de documents scientifiques de niveau recherche, publiés ou non, émanant des établissements d'enseignement et de recherche français ou étrangers, des laboratoires publics ou privés. 
Turbulence topology evolution in weakly turbulent premixed flames

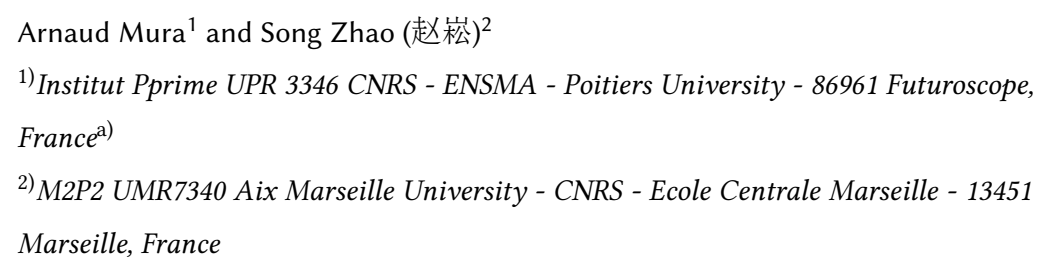

\section{Abstract}

In turbulent premixed flames, not only the isotropy of velocity fluctuations is altered by the thermal expansion effect, the dissipative structure of the turbulent flowfield and the flow topology are also deeply influenced by the flame. Considering the joint probability density function (JPDF) of the second and third invariants of the velocity gradient tensor (VGT) - or its traceless counterpart - is a classical way to educe the topology of turbulent flows at these smallest scales. These quantities are analyzed by considering direct numerical simulation databases of premixed flame kernel growth in homogeneous isotropic turbulence (HIT). Two conditions of turbulence-combustion interaction (TCI) are considered, which correspond to two distinct values of the Bray number. The analysis of the VGT shows that the propagating premixed flame and its associated density variations significantly modify the turbulence structure and flow topology. To understand this behavior as the flow interacts with the flame front, Lagrangian dynamics of the VGT and its invariants are studied by considering the conditional mean rate of change vectors. Special emphasis is thus placed on the Lagrangian evolution equations (LEE) of these invariants. To the best of the authors' knowledge, this is first time that such budgets are scrutinized in premixed combustion conditions. The pressure Hessian contribution to the VGT invariants transport equations is shown to be one of the leading-order terms in this evolution, making it critically important to the flow dynamics and turbulence structure.

Keywords: Combustion, flame, thermal expansion, turbulence, velocity gradient tensor

a)Electronic mail: arnaud.mura@ensma.fr 


\section{SCALAR MIXING AND TURBULENCE-SCALAR INTERACTIONS}

Scalar mixing in turbulent flows and turbulence-scalar interactions (TSI) are longstanding and challenging fluid dynamics issues that, for many years, have concentrated the research efforts of many world-renowned and prominent scientists. Professor E.E. O'Brien is one of them as a pioneer in the theory of turbulence using statistical approaches. As an example, O’Brien's early derivations of one- and two-point PDF transport equations, as well as scalar-gradient probability density function (PDF) budgets, were seminal contributions to the field of turbulence and turbulent reacting flows ${ }^{1-5}$. The consideration of such transport equations raises the issues of scalar mixing and conditional scalar dissipation rate modelling, which have been also investigated using direct numerical simulations ${ }^{6-8}$. The influence of isothermal or "cold" (i.e., non-exothermic) chemical reactions was analyzed ${ }^{9}$ as well as its possible impact on the spectral behaviour of reactive scalar in turbulent flows ${ }^{10}$. Finally, the concept of probability density functions was properly (i.e., rigorously) introduced within the large-eddy simulation framework leading to the filtered density function (FDF) transport equation for LES of turbulent reactive flows ${ }^{11}$. With all these developments and seminal findings, O’Brien has provided strong and solid bases for the development of PDF methods, which offer a general framework for turbulent combustion modelling ${ }^{12-15}$.

The scalar gradient (or dissipation rate) and two-point statistics (or structure function) were thus central to an important part of E.E. O'Brien's contributions. The possible influence of chemical reactions was also analyzed in many of them. However, in addition to the reactions that consume and produce chemical species, another important feature of combustion and flame is that turbulence-combustion interactions are made more complex by thermal expansion ${ }^{16,17}$. Indeed, the heat released by chemical reactions enforces a two-way coupling between the (turbulent) flow and the flame. Accordingly, the present study is focused on the impact of this thermal expansion and its consequence on the fluctuating velocity field and associated velocity gradient. The corresponding velocity gradient does not only drive the turbulence dissipation rate, i.e., the decay of the turbulent kinetic energy (TKE) as well as the velocity PDF evolution, it is also involved in the scalar gradient or scalar dissipation rate (SDR) evolution.

Indeed, if we consider a reference scalar $\xi(\boldsymbol{x}, t)$, the filtered or averaged transport equation of the corresponding SDR, which is defined as

$$
N_{\xi}=\mathscr{D} \frac{\partial \xi}{\partial x_{i}} \frac{\partial \xi}{\partial x_{i}}
$$


with $\mathscr{D}$ the scalar diffusivity, can be written as follows:

$$
\begin{aligned}
& \mathscr{L}\left(\overline{\rho N_{\xi}}\right)=\frac{\partial}{\partial t}\left(\overline{\rho N_{\xi}}\right)+\frac{\partial}{\partial x_{i}}\left(\overline{\rho u_{i} N_{\xi}}\right)-\frac{\partial}{\partial x_{i}}\left(\overline{\rho \mathscr{D} \frac{\partial N_{\xi}}{\partial x_{i}}}\right) \\
& =\text { TSI }-2 \rho \mathscr{D}^{2} \frac{\partial^{2} \xi}{\partial x_{i} \partial x_{j}} \frac{\partial^{2} \xi}{\partial x_{i} \partial x_{j}}+\text { OT }
\end{aligned}
$$

where $\mathscr{L}$ denotes a conservative transport operator. In the right-hand-side (RHS), TSI refers to the turbulence-scalar interaction term. The second term in the RHS of Eq. (2) is the SDR dissipation term, which does involve the curvature of the scalar field. Finally, OT represents other terms associated to scalar production-destruction. Depending on the scalar quantity $\xi$ under consideration, these terms may be associated to physical processes such as chemical reaction, vaporization, etc. In the following part of this study, attention will be focused on a scalar quantity that is used to represent the chemical evolution, i.e., the progress of chemical reactions. The corresponding variable is generally referred to as a progress variable, hereafter denoted $c(\boldsymbol{x}, t)$. This quantity can be assumed to be either the temperature $T(\boldsymbol{x}, t)$ or a species mass fraction $Y_{\alpha}(\boldsymbol{x}, t)$, normalized so that it is zero in the fresh reactants and unity in the burned gases, e.g., $c \equiv\left(Y_{\alpha}-Y_{\alpha}^{u}\right) /\left(Y_{\alpha}^{b}-Y_{\alpha}^{u}\right)$ with $Y_{\alpha}^{u}$ and $Y_{\alpha}^{b}$ the values of $Y_{\alpha}$ in fresh reactants and fully burned products, respectively. Its transport equation may be written as

$$
\mathscr{L}(\overline{\rho c})=\overline{\rho \dot{\omega}_{c}}
$$

with $\dot{\omega}_{c}$ its chemical production rate, while the corresponding SDR transport equation is given by $^{18-26}$

$$
\mathscr{L}\left(\overline{\rho N_{c}}\right)=\text { TSI }-2 \rho \mathscr{D}^{2} \frac{\partial^{2} c}{\partial x_{i} \partial x_{j}} \frac{\partial^{2} c}{\partial x_{i} \partial x_{j}}+2 \overline{\rho \mathscr{D} \frac{\partial c}{\partial x_{j}} \frac{\partial \dot{\omega}_{c}}{\partial x_{j}}}
$$

The TSI term - which appears in its right-hand-side - is defined as follows

$$
\mathrm{TSI}=-2 \rho \overline{\mathscr{D} \frac{\partial c}{\partial x_{i}} \frac{\partial u_{i}}{\partial x_{j}} \frac{\partial c}{\partial x_{j}}}
$$

an expression that does involve the velocity gradient tensor $A_{i j}=\partial u_{i} / \partial x_{j}$.

The present study is focused on the analysis of the influence of thermal expansion on the dynamics of the velocity gradient tensor (VGT) and associated flow topologies. The study of flow topology and dilatation effects is a topic of relevance to scalar mixing in turbulent flows ${ }^{27-30}$. However, studying the influence of the heat release-induced thermal expansion on turbulent flows is a difficult task that gathered an increasingly important research activity over recent 
years $^{16,17,21,23-27,31-36}$. It requires the use of sophisticated tools such as post-processing procedures based on geometrical analyses and streamline topology classification ${ }^{22,23,26,27,37}$ or flow velocity decomposition $31,36,38$. For instance, the recent studies of Sabelnikov et al. ${ }^{36,38}$ have been performed on the basis of the Helmholtz-Hodge decomposition of the velocity field $\boldsymbol{u}$ into solenoidal and potential components while Robin et al. ${ }^{31}$ introduced a splitting of the velocity field $u$ into two distinct contributions with the first component associated to turbulent motion $\boldsymbol{v}$ and its consequence on the flame surface and the second component $\boldsymbol{w}$ resulting from the acceleration induced by the local flame front. The corresponding kinetic energy of the flow, i.e., $\left(u_{i} u_{i}\right) / 2$, is redistributed and dissipated through pressure and viscous effects and, from a general viewpoint, whatever the strategy it seems difficult to get rid of the difficulty associated to the nonlocal action of the pressure field which is itself influenced by the thermal expansion. It is also noteworthy that the rate of dissipation of the kinetic energy $\varepsilon=\tau_{i j} A_{i j} / \rho$ and the components of the corresponding dissipation rate tensor $\varepsilon_{i j}$ are non linear functions of the VGT components and do not depend solely on solenoidal $\varepsilon_{i j}^{s}$ and potential $\varepsilon_{i j}^{p}$ contributions (or turbulent dissipation rate $\varepsilon_{i j}^{v v}$ and local flame dissipation rate $\left.\varepsilon_{i j}^{w w}\right)$. They do involve also mixed or cross terms the contributions of which are non-negligible ${ }^{31,38}$. From the above discussion, it is quite clear that the VGT is a central issue for the study of turbulence and mixing in turbulent premixed flames. The VGT can be characterized through its invariants, namely the three coefficients of its characteristic polynomial. Lagrangian evolution equations (LEE) are written for these invariants. Direct numerical simulations databases are used to analyze the various contributions (interaction between invariants, pressure Hessian, baroclinic effect, and viscous processes) and the possible impact of thermal expansion.

The manuscript is organized as follows: the next section (Section II) settles the fundamental bases of the analysis and describes the associated mathematical background. It includes the presentation of the LEE for the three invariants of the VGT. The DNS databases are then briefly described in Section III. The post-processed results and their analyses are gathered in Section IV. Finally, the paper ends with a conclusion section (Section V) where the main outcomes of the present study are summarized. 


\section{CURRENT STATE OF KNOWLEDGE AND THEORETICAL BACKGROUND}

The present study is focused on the analysis of the influence of thermal expansion on the dynamics of the velocity gradient tensor (VGT). The VGT can be characteristized through its invariants, namely the three coefficients of its characteristic polynomial. In fact the influence of the thermal expansion on the large-scale features of the turbulent velocity field has been early emphasized in the pioneering works of Libby and Bray and their coworkers ${ }^{39,40}$, leading to refined closures for turbulent transport in flames able to reproduce the flame-generated turbulence phenomena ${ }^{41-43}$. The corresponding studies have required significant efforts devoted to the modelling of pressure terms in the Reynolds stresses and scalar fluxes transport equations ${ }^{42,43}$, with some specific insights gained into their relationships with the heat release rate. Most of these studies have been concerned with the description of the large-scale characteristics of the turbulent reactive flows. Following the recent achievements obtained in the application of large-eddy simulations to reactive flows, the analysis of thermal expansion effects has been more recently focused on the study of the smallest scales ${ }^{21,24-26,44}$.

As emphasized in the previous section, the present study is focused on the analysis of the velocity gradient tensor $\boldsymbol{A}$, the component of which are given by

$$
A_{i j} \equiv \frac{\partial u_{i}}{\partial x_{j}}=S_{i j}+W_{i j}
$$

with $S$ its symmetric part

$$
S_{i j} \equiv \frac{1}{2}\left(A_{i j}+A_{j i}\right)=\frac{1}{2}\left(\frac{\partial u_{i}}{\partial x_{j}}+\frac{\partial u_{j}}{\partial x_{i}}\right)
$$

and $\boldsymbol{W}$ its antisymetric part

$$
W_{i j} \equiv \frac{1}{2}\left(A_{i j}-A_{j i}\right)=\frac{1}{2}\left(\frac{\partial u_{i}}{\partial x_{j}}-\frac{\partial u_{j}}{\partial x_{i}}\right) .
$$

The eigen values of the VGT, i.e., $\lambda_{1}, \lambda_{2}$ and $\lambda_{3}$ (in decreasing order $\lambda_{1} \geq \lambda_{2} \geq \lambda_{3}$ ) are the roots of its characteristic equation

$$
\operatorname{det}[\boldsymbol{A}-\lambda \boldsymbol{I}]=0.0,
$$

while the invariants $P, Q$, and $R$ of the VGT are defined as the normalized coefficients of this characteristic equation

$$
\lambda^{3}+P \lambda^{2}+Q \lambda+R=0 .
$$


They can be expressed as ${ }^{45}$

$$
\begin{aligned}
& P=-\operatorname{tr}[\boldsymbol{A}]=-A_{k k}, \\
& Q=\frac{1}{2}\left(P^{2}-\operatorname{tr}[\boldsymbol{A} \boldsymbol{A}]\right)=\frac{1}{2}\left(P^{2}-A_{i j} A_{j i}\right), \\
& R=\frac{1}{3}\left(-P^{3}+3 P Q-\operatorname{tr}[\boldsymbol{A} \boldsymbol{A} \boldsymbol{A}]\right)=\frac{1}{3}\left(-P^{3}+3 P Q-A_{i j} A_{j k} A_{k i}\right) .
\end{aligned}
$$

Their deviatoric counterparts may also be defined from the traceless part of the VGT tensor $A^{*}$

$$
A_{i j}^{*} \equiv A_{i j}-\frac{\theta}{3} \delta_{i j}
$$

with $\delta_{i j}$ the Kronecker tensor and $\theta \equiv \operatorname{tr}[\boldsymbol{A}]=A_{k k}=-P$ the dilatation-rate, i.e., the trace of $\boldsymbol{A}$ Similarly, the symmetric and antisymmetric parts of $\boldsymbol{A}^{*}$ are denoted as $\boldsymbol{S}^{*}$ and $\boldsymbol{W}^{*}$, respectively, and the invariants of $\boldsymbol{A}^{*}$ can be expressed as ${ }^{46}$

$$
\begin{aligned}
P^{*} & =-A_{k k}^{*} \equiv 0, \\
Q^{*} & =-\frac{1}{2} A_{i j}^{*} A_{j i}^{*}, \\
R^{*} & =-\frac{1}{3} A_{i j}^{*} A_{j k}^{*} A_{k i}^{*} .
\end{aligned}
$$

From the above set of definitions, the Lagrangian evolution equations (LEE) of the VGT invariants can be derived ${ }^{30,47}$. The starting point of such a derivation is the transport equation of the VGT component, which is given by ${ }^{47-50}$

$$
\dot{A}_{i j} \equiv \frac{D A_{i j}}{D t}=\frac{\partial A_{i j}}{\partial t}+u_{k} \frac{\partial A_{i j}}{\partial x_{k}}=-\frac{\partial}{\partial x_{j}}\left(\frac{1}{\rho} \frac{\partial p}{\partial x_{i}}\right)+\frac{\partial}{\partial x_{j}}\left(\frac{1}{\rho} \frac{\partial \tau_{i k}}{\partial x_{k}}\right),
$$

where $D / D t$ denotes the substantial (or total) derivative, $p$ is the pressure, and $\tau_{i j}=2 \mu S_{i j}^{*}$ is the viscous stress tensor.

The LEE of the three invariants $(P, Q$, and $R)$ write as follows:

$$
\begin{aligned}
& \dot{P} \equiv \frac{D P}{D t}= \underbrace{\left(P^{2}-2 Q\right)}_{\mathrm{I}_{P}}+\underbrace{\mathscr{H}_{i i}^{p}}_{\mathrm{II}_{P}}+\underbrace{\mathscr{H}_{i i}^{b}}_{\mathrm{III}_{P}}+\underbrace{\left(-\mathscr{T}_{i i}\right)}_{\mathrm{IV}_{P}}, \\
& \dot{Q} \equiv \frac{D Q}{D t}=\underbrace{(P Q-3 R)}_{\mathrm{I}_{Q}}+\underbrace{\left(P \mathscr{H}_{i i}^{p}+A_{i j} \mathscr{H}_{j i}^{p}\right)}_{\mathrm{II}_{Q}}+\underbrace{\left(P \mathscr{H}_{i i}^{b}+A_{i j} \mathscr{H}_{j i}^{b}\right)}_{\mathrm{III}_{Q}}+\underbrace{\left(-P \mathscr{T}_{i i}-A_{i j} \mathscr{T}_{j i}\right)}_{\mathrm{IV}_{Q}}, \\
& \dot{R} \equiv \frac{D R}{D t}=\underbrace{(P R)}_{\mathrm{I}_{R}}+\underbrace{\left(Q \mathscr{H}_{i i}^{p}+P A_{i j} \mathscr{H}_{j i}^{p}+A_{i k} A_{k j} \mathscr{H}_{j i}^{p}\right)}_{\mathrm{II}_{R}}+\underbrace{\left(Q \mathscr{H}_{i i}^{b}+P A_{i j} \mathscr{H}_{j i}^{b}+A_{i k} A_{k j} \mathscr{H}_{j i}^{b}\right)}_{\mathrm{II}_{R}} \\
&+\underbrace{\left(-Q \mathscr{T}_{i i}-P A_{i j} \mathscr{T}_{j i}-A_{i j} A_{j k} \mathscr{T}_{k i}\right)}_{\mathrm{IV}_{R}},
\end{aligned}
$$


where the first terms (I) of the RHS of these equations are associated to mutual interactions between the invariants. The second (II) and third terms (III) correspond to pressure Hessian and baroclinic effects ${ }^{49}$, respectively. Finally, the fourth terms (IV) represent viscous contributions.

The two contributions involved in the pressure terms, i.e., pressure Hessian and baroclinic terms, are defined as follows

$$
\mathscr{H}_{i j} \equiv \frac{\partial}{\partial x_{j}}\left(\frac{1}{\rho} \frac{\partial p}{\partial x_{i}}\right)=\frac{1}{\rho} \frac{\partial^{2} p}{\partial x_{i} \partial x_{j}}+\left(-\frac{1}{\rho^{2}} \frac{\partial \rho}{\partial x_{j}} \frac{\partial p}{\partial x_{i}}\right) \equiv \mathscr{H}_{i j}^{p}+\mathscr{H}_{i j}^{b}
$$

while the viscous term is given by

$$
\mathscr{T}_{i j} \equiv \frac{\partial}{\partial x_{j}}\left(\frac{1}{\rho} \frac{\partial \tau_{i k}}{\partial x_{k}}\right)
$$

At this level, it must emphasized that similar LEE can be readily derived for the invariants of the traceless VGT $\boldsymbol{A}^{*}$, as it is shown in Appendix A. The traceless VGT is indeed often considered to analyze non-reactive turbulent flows featuring compressibility and/or dilatational effects $^{29,30,46,51}$. At this level, it must also be emphasized that some similarities are expected between the present reactive flows featuring local premixed flame fronts and other turbulent flows featuring sharp density variations, e.g., shock waves, detonations, or two-phase flows featuring mass transfer (evaporation). Such a claim is supported by recent analyses of turbulent mixing in compressible flows with shock waves ${ }^{29,51}$, where significant increases of the probability to find non-focal streamline topologies have been put into evidence. Thus, the interest of the present analysis may exceed the restricted scope of turbulent premixed combustion. From a general point of view, the investigation of flow topologies in such variable-density flows remains rather limited in comparison with that of incompressible turbulence. In this respect, the joint statistics of the second and third invariants of the traceless VGT (i.e., $Q^{*}$ and $R^{*}$ ) in turbulent premixed flames has been only very recently reported by ourselves ${ }^{52}$. Specific thermal expansion effects were put into evidence and one of the objectives of the present study is to proceed with a refined and comprehensive analysis of the corresponding effects based on the LEE. To the best of the authors' knowledge, this is the first time such an analysis of the LEE is conducted in turbulent premixed flames. In the next sections, the DNS databases retained to proceed with this analysis are presented and the various terms present in the RHS of the LEE of both the traceless VGT $\boldsymbol{A}^{*}$ and the VGT $\boldsymbol{A}$ are scrutinized. 


\section{DNS DATABASES OF FLAME KERNEL GROWTH IN WEAKLY TURBULENT FLOWS}

The detailed analysis of LEE (i.e., the dynamics of the VGT invariants) is conducted on the basis of databases issued from the direct numerical simulation (DNS) of flame kernel growth in initially homogeneous isotropic turbulence (HIT). The corresponding set of data has been generated with the Asphodele solver ${ }^{53}$. This solver, the main features of which have been already presented elsewhere ${ }^{53}$, is based on a dilatable low-Mach number framework. Spatial derivatives are evaluated on regular meshes by making use of high-order precision centered finite difference (FD) schemes while time integration is carried out with a minimal storage third-order explicit Runge-Kutta (RK) scheme. The pressure-velocity coupling is handled within a standard projection-correction framework. We consider lean mixtures of iso-octane $\mathrm{C}_{8} \mathrm{H}_{18}$ and air featuring an equivalence ratio value $\Phi=0.8$. The temperature in the fresh reactants is $T_{u}=700 \mathrm{~K}$ and the pressure is $P_{0}=5 \mathrm{bar}$. The corresponding values of the laminar flame speed is approximately $S_{L}^{0}=70 \mathrm{~cm} / \mathrm{s}$. Two distinct direct numerical simulations of flame kernel development are performed in three-dimensional computational domains of $4.0 \times 4.0 \times 4.0 \mathrm{~mm}^{3}$ featuring 256 grid points in each direction. Periodic boundary conditions are considered along each of these three directions. The turbulence characteristics for the two cases are different and the values of the turbulent velocity fluctuation $\left(u_{\mathrm{RMS}}\right)$, integral length scale $\left(l_{t}\right)$, Kolmogorov length scale $\left(\eta_{K}\right)$, and turbulent Reynolds number $\left(\operatorname{Re}_{t}=u_{\mathrm{RMS}} l_{t} / v_{u}\right)$ of the corresponding initial turbulent flowfields are gathered in Table I.

Table I. Characteristics of the initial HIT for both simulation cases

\begin{tabular}{ccccc}
\hline \hline Simulation & $u_{\mathrm{RMS}}(\mathrm{m} / \mathrm{s})$ & $l_{t}(\mathrm{~mm})$ & $\eta_{K}(\mu \mathrm{m})$ & $\mathrm{Re}_{t}$ \\
$\mathrm{~F} 1$ & 0.5 & 0.6 & 57 & 23 \\
$\mathrm{~F} 2$ & 1.0 & 0.4 & 30 & 31 \\
\hline \hline
\end{tabular}

The two conditions are denoted F1 and F2, and the RMS of velocity fluctuations is approximately two times larger in the second case. Based on the unity flame Reynolds number assumption (i.e., $\operatorname{Re}_{f}=S_{L}^{0} \delta_{L}^{0} / v_{u}=1$, with $v_{u}$ the molecular viscosity in the fresh mixture), the values of the Damköhler and Karlovitz numbers can be evaluated. These values may be used to delineate 
Table II. Turbulence-combustion interaction characteristics for both simulated cases

\begin{tabular}{|c|c|c|c|c|c|}
\hline Simulati & ${ }_{t} / \delta_{L}^{0}(-)$ & $u_{\mathrm{RMS}} / S_{L}^{0}$ & $\mathrm{Da}(-)$ & $\mathrm{Ka}(-)$ & $\mathrm{N}_{B}(-)$ \\
\hline F1 & 32.8 & 0.71 & 44.9 & 0.11 & 1.65 \\
\hline F2 & 21.9 & 1.43 & 14.9 & 0.37 & 0.83 \\
\hline
\end{tabular}

the combustion regimes in a standard combustion diagram ${ }^{54-56}$. Note that the value of the ratio $\tau S_{L}^{0} /\left(2 u_{\mathrm{RMS}}\right)$, hereafter referred to as the Bray number $\mathrm{N}_{B}{ }^{25,31,57}$ is also reported in Table II. The condition F1 corresponds to a quasi-laminar flame that lies in the wrinkled flame regime. The values of $\mathrm{N}_{B}$ reported in Table 1 confirm that it is dominated by thermal expansion effects. The computed values of the Damköhler number $\mathrm{Da}=\left(l t / u_{\mathrm{RMS}}\right) \times\left(S_{L}^{0} / \delta_{L}^{0}\right)$ are significantly larger than unity in both cases; Da is approximately forty-five for flame F1 and fifteen for flame F2. The turbulent premixed flame F2 lies within the corrugated flamelets regime. However, the value of the Karlovitz number Ka in this case is approximately four times larger than its counterpart in case F1, see Table II. The two cases F1 and F2 can be relevant to small-scale Laboratory flames such as those studied for micro gas turbine applications ${ }^{58,59}$.

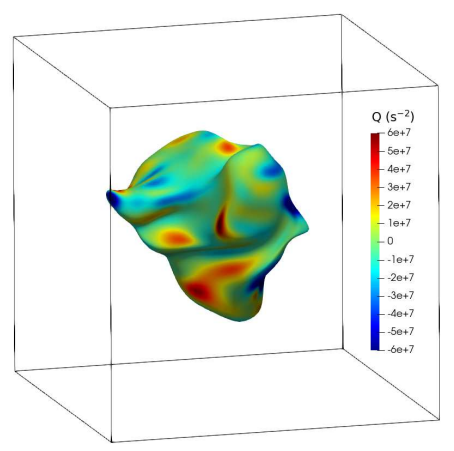

(a) $t^{*}=2.0$

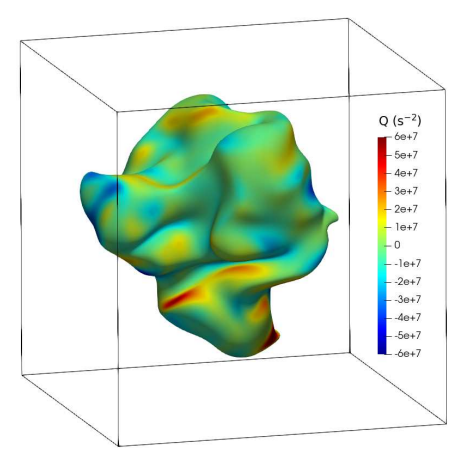

(b) $t^{*}=3.0$

Figure 1. Instantaneous snapshots of the turbulent flame kernels F2 at times $t^{*}=2.0$ (a) and $t^{*}=3.0$ (b): iso-contour $c=0.01$ colored by the second invariant $Q$.

The DNS databases correspond to freely-evolving turbulence, no artificial forcing has been considered. The values reported in Table I apply to initial values of the turbulent velocity fields 


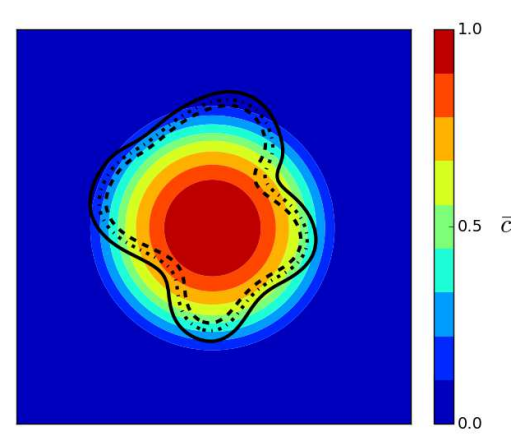

(a) Case F1

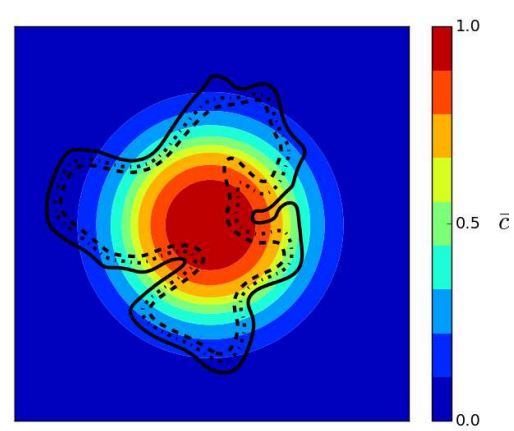

(b) Case F2

Figure 2. Field (in colors) of the averaged progress variable $\bar{c}$ at $t^{*}=3.0$ together with three instantaneous progress variable iso-lines issued from a cut-plane of the computational domain (solid line: $c=0.01$, dot-dashed line: $c=0.50$, dashed line: $c=0.95$ ). Case F1 on the left (a) and case F2 on the right (b).

that are initialized at time $t=0.0$ with a Passot-Pouquet spectrum ${ }^{60}$ and the details of this initialization procedure can be found elsewhere ${ }^{52}$. Instantaneous snapshots of the three-dimensional turbulent flame kernels are displayed in Fig. 1. The displayed flame contour corresponds to an iso-value of the progress variable $c$, which is defined according to

$$
c \equiv \frac{Y_{\mathrm{C}_{8} \mathrm{H}_{18}}-Y_{\mathrm{C}_{8} \mathrm{H}_{18}}^{u}}{Y_{\mathrm{C}_{8} \mathrm{H}_{18}}^{b}-Y_{\mathrm{C}_{8} \mathrm{H}_{18}}^{u}},
$$

with $Y_{\mathrm{C}_{8} \mathrm{H}_{18}}$ the mass fraction of iso-octane, $Y_{\mathrm{C}_{8} \mathrm{H}_{18}}^{u}$ and $Y_{\mathrm{C}_{8}}^{b} \mathrm{H}_{18}$ the mass fraction values of isooctane in the fresh reactants and fully burnt products, respectively. The corresponding isocontour value is $c=0.01$. It is colored by the value of the second invariant $Q$ of the VGT and it is plotted for two distinct values of the non-dimensional time $t^{*}=t / \tau_{f}$, i.e., the physical time $t$ normalized by the flame transit time $\tau_{f}=\delta_{L}^{0} / S_{L}^{0}$. The corresponding set of figures clearly confirms that the turbulent flame F2 displays significant levels of curvature and wrinkling. In the following, unless otherwise stated, the results that are presented have been obtained at $t^{*}=3.0$. The flame-turbulence interaction is further illustrated in Fig. 2 that displays three instantaneous iso-values of the progress variable $c$ superimposed on the mean progress variable field $\bar{c}$. These mean values are obtained by averaging the progress variable field at time $t$ on a spherical shell around the radius $r \equiv\left|\boldsymbol{x}-\boldsymbol{x}_{\boldsymbol{c}}\right|$, where $\boldsymbol{x}_{\boldsymbol{c}}$ denotes the center of the computational domain. Thus, 
averaged fields of the progress variable $\bar{c}$ are obtained based on a radial symmetry assumption, which is also often retained to analyze experimental databases ${ }^{61}$. In this figure, the depicted iso-lines of the progress variable correspond to $c=0.01, c=0.95$, and $c=0.50$. The first two are used to delineate the flame boundaries, while the last one corresponds approximately the maximum derivative of the heat release rate (HRR) with respect to the progress variable, as determined from the laminar premixed flame of reference. These isolines have been extracted from the computational data in the medium horizontal plane $(x, y)$, i.e., at $z=0$. From this figure, it is noteworthy that the spacing (i.e., the distance) between two consecutive iso-levels of $\bar{c}$ is larger in case F2 than in case F1. The consideration of instantaneous progress variable iso-lines undoubtedly confirms that, for flame F2, there is indeed non-negligible local thickening of the preheat zone $\mathrm{e}^{20,62}$.

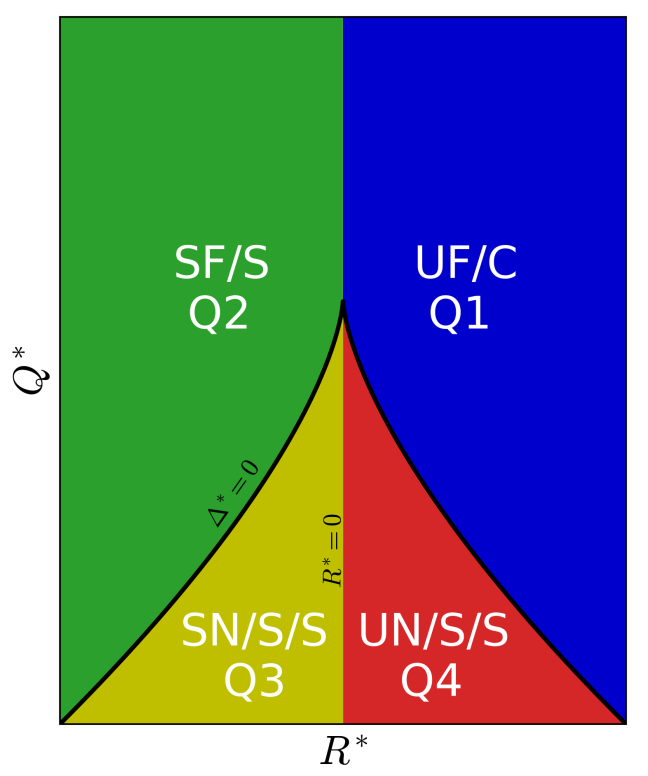

Figure 3. Local streamline topology classification in the second and third VGT invariants sub-space. 


\section{ANALYSIS OF THE LAGRANGIAN EVOLUTION EQUATIONS (LEE) OF THE VGT INVARIANTS}

The main objective of the present study is to analyze the heat-release-induced thermal expansion effects on the VGT. Accordingly, attention is focused on case F1, which is associated to the largest value of the Bray number. Similar (but less important) effects are observed with the data gathered in case F2. For the sake of conciseness and in order to avoid many repetitions, the study of the flame F2 has been therefore summarized in Appendix C.

In a first step of the analysis, the invariants of the traceless VGT are analyzed. Four distinct regions can be delineated in the map of the third and second invariants $\left(R^{*}, Q^{*}\right)$ by considering the iso-line $R^{*}=0.0$ and the two curves originating from the origin $\left(R^{*}=0.0, Q^{*}=0.0\right)$, curves that gather solutions to $\Delta^{*} \equiv(27 / 4) R^{* 2}+Q^{* 3}=0.0$, i.e., zero values of the discriminant of the characteristic polynomial of $\boldsymbol{A}^{*}$. According to the terminology of Perry and Chong ${ }^{63,64}$, the four regions mentioned above correspond to unstable-focus compressing (UF/C), stable-focus stretching (SF/S), stable-node saddle-saddle (SN/S/S), and unstable-node saddle-saddle (UN/S/S) from the top right-hand corner to the bottom right-hand corner (counter-clockwise). These various regions are depicted in Fig. 3. The topologies above the zero-discriminant lines, i.e., UF/C and $\mathrm{SF} / \mathrm{S}$, are spiraling in nature and are often referred to as focal topologies whereas the topologies below these lines, i.e. SN/S/S and UN/S/S, do not spiral about a focus and are therefore termed as non-focal topologies. These four regions will be hereafter denoted by quadrants $Q_{1}, Q_{2}, Q_{3}$, and $Q_{4}$, respectively.

In this respect, it has been early shown that, in standard incompressible turbulence, e.g., homogeneous isotropic turbulence (HIT), there is an increased probability of points where $R>0$ and $Q<0$ along the so-called Vieillefosse tail ${ }^{65}$. The joint PDF of $\left(R^{*}, Q^{*}\right)$ has a tear-drop shape with high probability levels associated to the SF/S topology. This is in contrast with the behaviour observed for the invariants of the traceless VGT across a laminar premixed flame. Indeed, it can be shown (see Appendix B) that, in one-dimensional laminar premixed flame structures, the invariants pairs $\left(R^{*}, Q^{*}\right)$ evolve along the left branch of the zero discriminant curve $\Delta^{*}=27 / 4 R^{* 2}+Q^{* 3}=0.0$. This is a direct outcome of the flow acceleration that is induced by thermal expansion. Thus, provided that the local velocity gradient remains dominated by the local acceleration that takes place across flamelets ${ }^{25}$ (i.e., for sufficiently large values of the Bray number), it is expected that, in turbulent premixed flames, the SN/S/S topology (quadrant $Q_{3}$ ) 

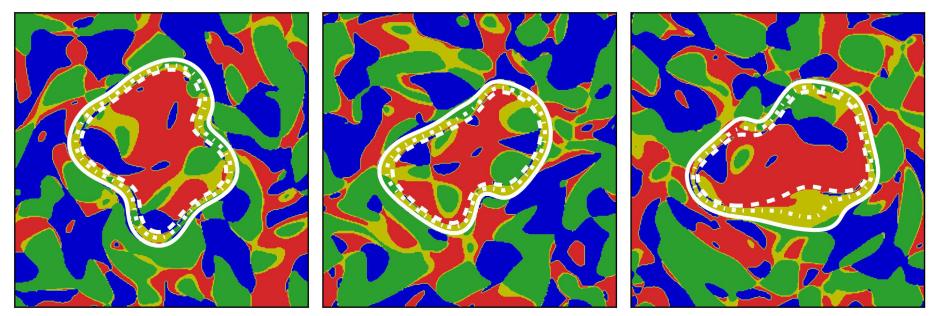

(a) Median cut-planes along $x$-, $y$-, and $z$-direction

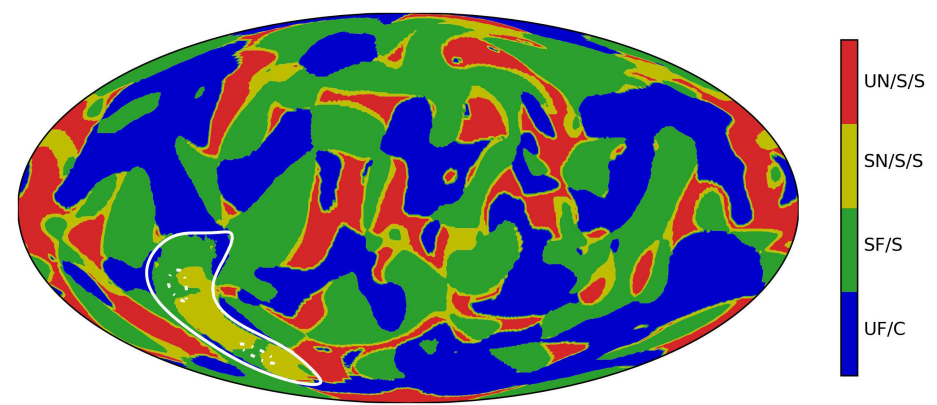

(b) Mollweide projection of the sphere associated to the iso-surface $\bar{c}=0.01$

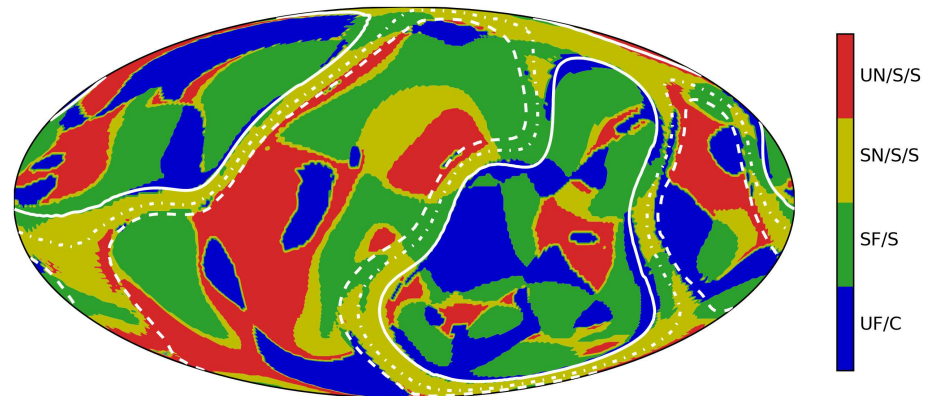

(c) Mollweide projection of the sphere associated to the iso-surface $\bar{c}=0.50$

Figure 4. Illustration of the flow topology in median cut-planes along $x$-, $y$-, and $z$-direction (a), on the surface associated to the iso-contour $\bar{c}=0.01$ of the mean progress variable (b), and on the surface associated to the iso-contour $\bar{c}=0.50$ of the mean progress variable (c) for simulation F1. Solid white line: iso-contour $c=0.01$, dot-dashed line: $c=0.50$, dashed line: $c=0.95$. 
displays increased probability levels in the flame region.

Figures 4 and 5 display an illustration of the flow topology obtained in median cut-planes along $x$-, $y$-, and $z$-directions, for simulations F1 and F2, respectively. However, with the objective of analyzing thermal expansion and associated density variation effects - effects which are taking place within the thickness the turbulent flame brush (where the mean progress variable is larger than zero and smaller than unity) - it is quite standard to display variations on surfaces associated to iso-values of the mean progress variable $\bar{c}$. Therefore, the flow topology is also displayed on sphere surfaces associated to iso-contours $\bar{c}=0.01$ (i.e., at the leading edge of the turbulent premixed flame) and $\bar{c}=0.50$. At this level, it seems worth precising that the corresponding results, collected on the spherical surfaces, have been displayed by using the Mollweide projection method. The Mollweide projection is an equal-area pseudo-cylindrical projection procedure that has been often used to produce maps of the earth surface. It trades accuracy of angle and shape for accuracy of proportions in area. It is therefore suited to illustrate global distributions such as those reported in Figs. 4 and 5. The projection transforms latitude and longitude coordinates, i.e., $(\phi, \lambda)$, into two-dimensional cartesian coordinates $x=(2 \sqrt{2} / \pi) \lambda \cos \theta$ and $y=\sqrt{2} \sin \theta$, where $\theta$ is an auxiliary angle defined by $2 \theta+\sin 2 \theta=\pi \sin \phi$. Figure 4 shows that, in the flame region, the probability of $\mathrm{SF} / \mathrm{S}$ (quadrant $Q_{2}$, green color) is decreased compared to its level in the fresh reactants region. This figure also confirms undoubtedly that the probability of SN/S/S (quadrant $Q_{3}$, yellow color) is increased in the flame region. Similar changes are observed in Fig. 5 but they are less marked, a behaviour that is fully consistent with the value of the Bray number $\mathrm{N}_{B}$, which is approximately two times smaller for case F2 compared to case F1, see Table II.

The conclusions of the above discussion are more quantitatively assessed in Fig. 6, which displays the joint PDF of $\left(R^{*}, Q^{*}\right)$ obtained in the fresh reactants (left column), in the flame region (middle column), and in the burned products (right column), for case F1 (top) and case F2 (bottom). These values of the invariants are normalized with the squared vorticity averaged over the whole computational domain, hereafter denoted by $\left\langle\omega^{2}\right\rangle$. In the fresh reactants (left column of Fig. 6), standard teardrop shapes are recovered for the joint PDFs in both conditions (case F1 and case F2). They are associated to significant probability levels of SF/S (quadrant $Q_{2}$ ) and UN/S/S (quadrant $Q_{4}$ ) topologies. Obtaining, in the fresh reactants, such joint PDF statistics similar to those observed in constant density flows ${ }^{66}$ is fully consistent with the results previously documented by Cifuentes et al. ${ }^{27}$ and Wacks et al. ${ }^{37}$. This is however in contrast with the behaviour observed inside the flame brush (middle column of Fig. 6), where there is a significant increase 

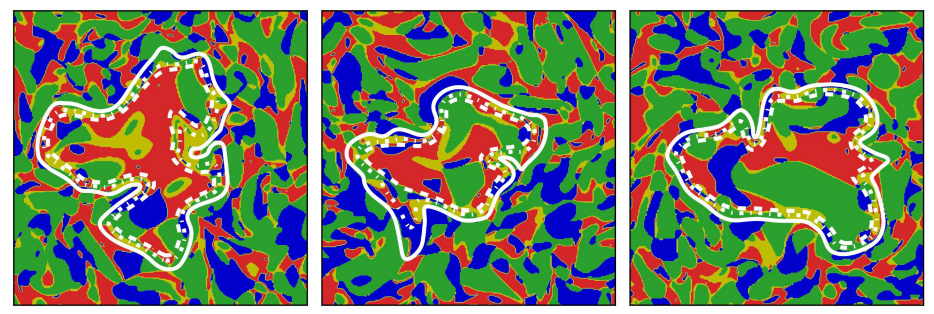

(a) Median cut-planes along $x$-, $y$-, and $z$-direction

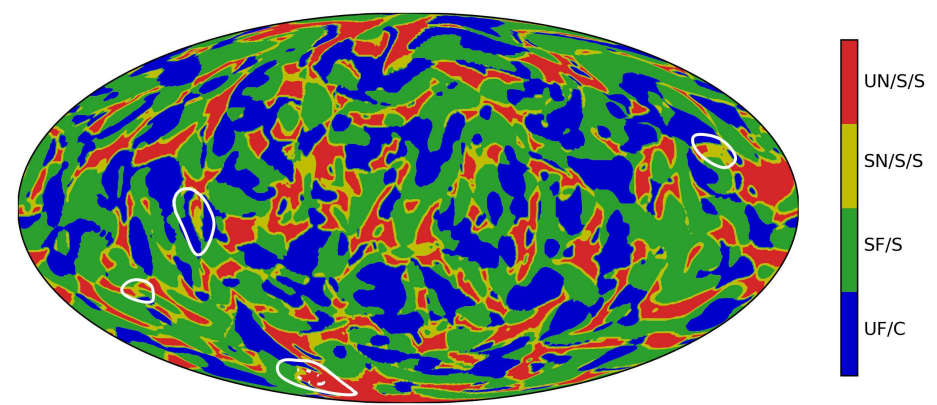

(b) Mollweide projection of the sphere associated to the iso-surface $\bar{c}=0.01$

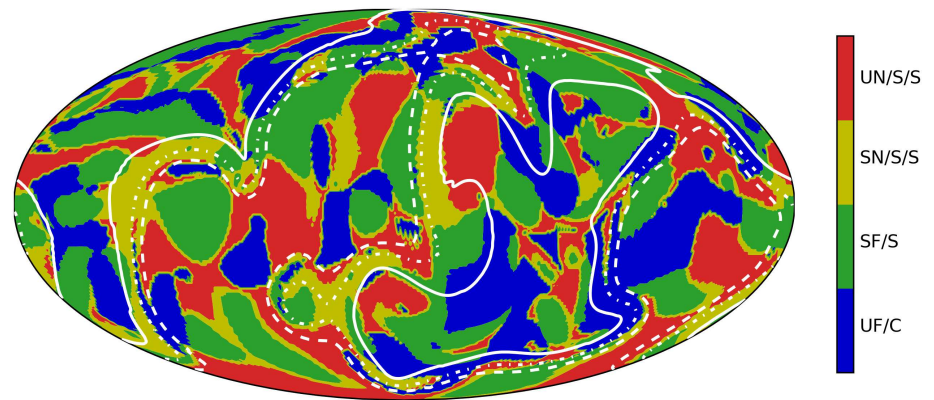

(c) Mollweide projection of the sphere associated to the iso-surface $\bar{c}=0.50$

Figure 5. Illustration of the flow topology in median cut-planes along $x$-, $y$-, and $z$-direction (a), on the surface associated to the iso-contour $\bar{c}=0.01$ of the mean progress variable (b), and on the surface associated to the iso-contour $\bar{c}=0.50$ of the mean progress variable (c) for simulation F2. Solid white line: iso-contour $c=0.01$, dot-dashed line: $c=0.50$, dashed line: $c=0.95$. 

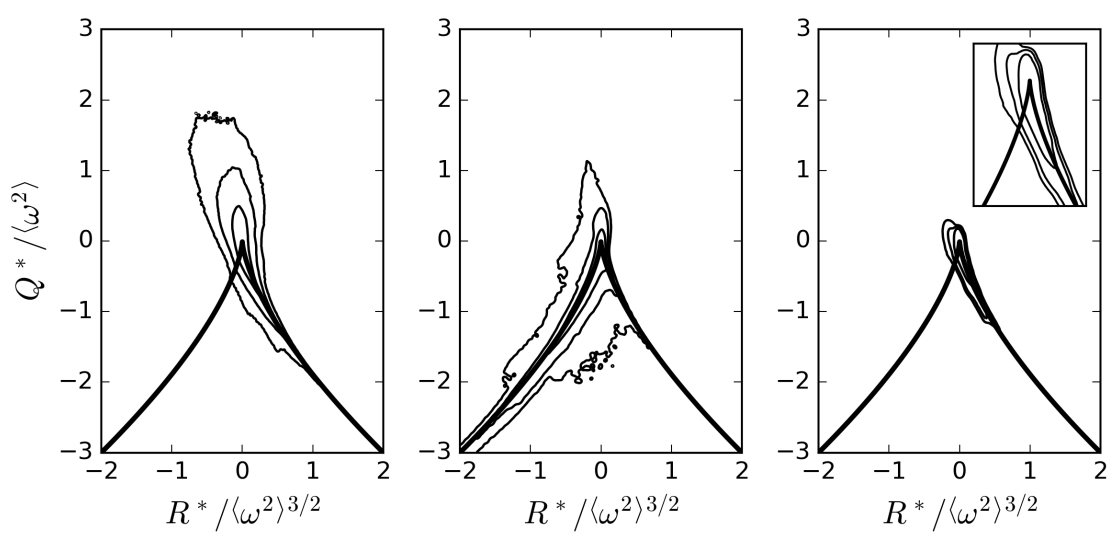

(a) Case F1
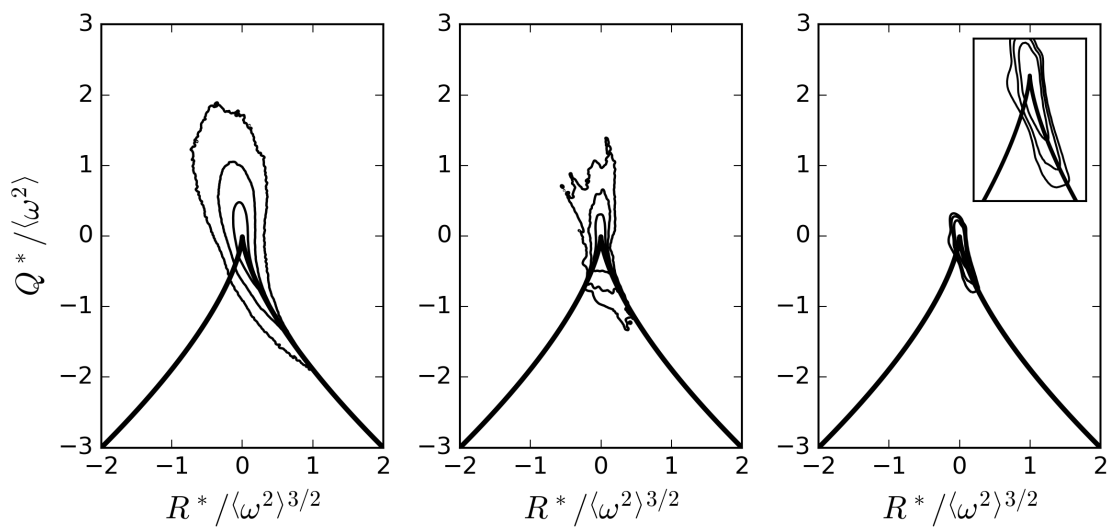

(b) Case F2

Figure 6. Iso-contours of the joint PDF of the third and second invariants $\left(R^{*}, Q^{*}\right)$ of the traceless VGT in case F1 (a) and case F2 (b). Left column: fresh reactants $(c<0.01)$, middle: flame region $(0.01<c<0.95)$, right: burnt gases $(c>0.95)$. The three thin lines are the iso-contours of $\log _{10}(\mathrm{JPDF})$ equals to $-2.0,-1.0$, and 0.0 . 

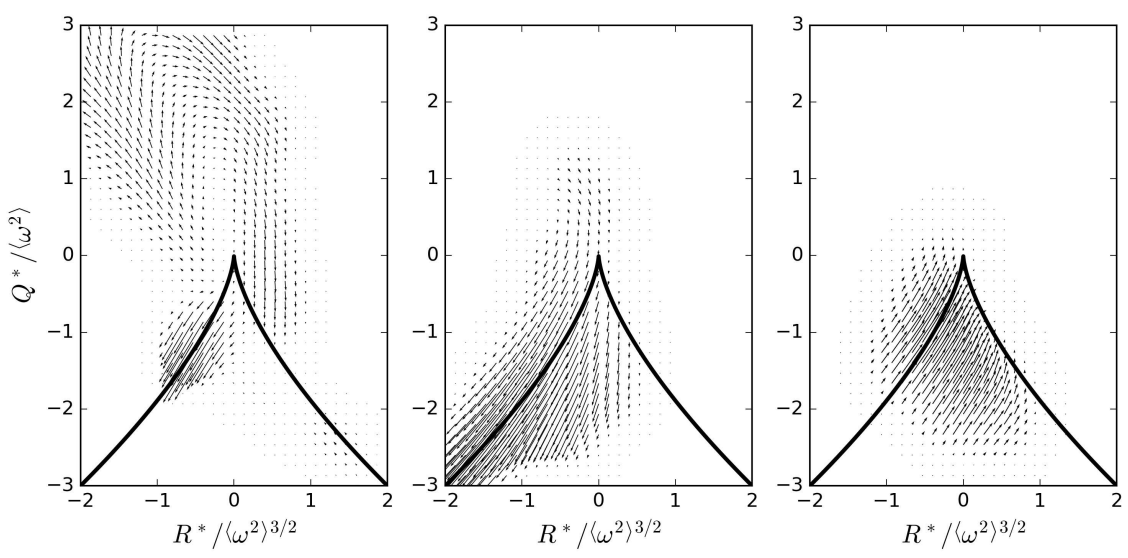

(a) Case F1
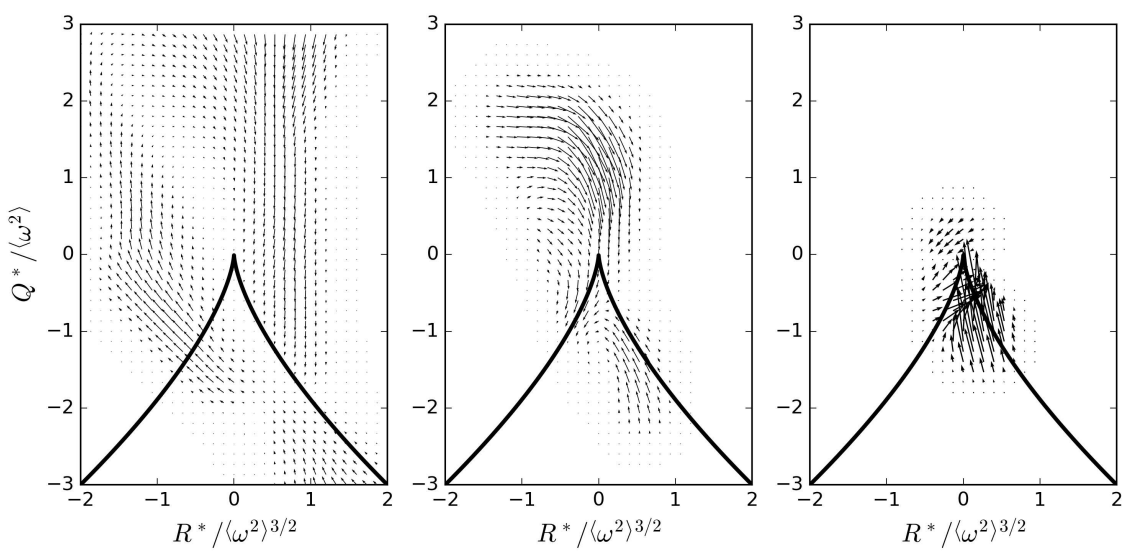

(b) Case F2

Figure 7. Conditional mean vectors in the $\left(R^{*}, Q^{*}\right)$ invariants plane for case F1 (a) and case F2 (b). Left column: $c<0.01$ (fresh reactants), middle column: $0.01<c<0.50$, right column: $0.50<c<0.95$.

in the probability associated to SN/S/S topologies especially in the vicinity of the left branch of the zero discriminant iso-line $\left(\Delta^{*}=0.0\right)$. This tendency is quite pronounced in case F1 whereas it remains almost negligible in case F2. In the burnt gases (right column of Fig. 6), the ranges of variations of both quantities $R^{*}$ and $Q^{*}$ are significantly decreased by viscous effects. It is, 
however, noteworthy that the joint PDFs tend to relax towards standard tear-drop shapes similar to those already observed in the fresh reactants, although the SF/S topology seems to be quite less relevant in the burnt gases.

The Lagrangian evolution of the turbulence and flow topology are examined in further detail by considering the conditional mean rates of change of $R^{*}$ and $Q^{*}$ in the invariants plane ${ }^{67}$. The corresponding rates of change are used to form a vector at each point in this plane. The resulting trajectories are followed to get some new insights into the role of thermal expansion on turbulence modulation. The normalized conditional mean vectors $\left(\left(D R^{*} / D t\right) /\left\langle\omega^{2}\right\rangle^{3 / 2},\left(D Q^{*} / D t\right) /\left\langle\omega^{2}\right\rangle\right)$ are displayed in Fig. 7 for various regions of the turbulent reacting flows. The vectors obtained in the fresh reactants are rather similar to those previously documented for homogeneous isotropic turbulence (HIT) or other incompressible/compressible canonical turbulent flows ${ }^{47,67}$. Indeed, in such canonical turbulent flows, the conditional mean vectors (CMVs) exhibit a circulating behavior in the $(R, Q)$ plane around the origin and in the clockwise direction, indicating that the flow evolves from SF/S to UF/C, UN/S/S, SN/S/S and then back to SF/S on average. Such a circulating behavior is relevant to the Lagrangian dynamics in fully developed turbulence that maintains the tear-drop shape of the joint statistical distribution of $(R, Q)$. However, although the main part of the CMV field seems to contribute to maintain the tear-drop shape of the joint PDF of the third and second invariants, it exhibits also a non-negligible contribution that drives this joint PDF from the origin point towards the left branch of the line $\Delta^{*}=0.0$. This behaviour is quite remarkable for case F1, which is associated to the largest value of the Bray number. Moreover, as shown in the middle column of Fig. 7, this phenomenon is more significant in the pre-heat zone $(0.01<c<0.50)$ where the most important part of the $\left(D R^{*} / D t, D Q^{*} / D t\right)$ vector is oriented towards the left branch of the zero-discriminant line. This is in contrast to the statistics gathered in the region where chemistry is more active $(0.50<c<0.95)$, which is displayed in the right column of Fig. 7: in this region, the joint $\operatorname{PDF}\left(R^{*}, Q^{*}\right)$ is relaxed towards the origin, leading to a much smaller tear-drop shape, as previously shown in Fig. 6.

The various contributions that appears in the RHS of the LEE of the second and third invariants of the traceless VGT, see Eqs. (A3), are now analyzed in details for case F1. The interactions between invariants, i.e., $\left(\mathrm{I}_{R^{*}}, \mathrm{I}_{Q^{*}}\right)$, are plotted in Fig. 8(a). On the fresh gas side (left column), the effects of this term is quite similar to the whole contribution $\left(D R^{*} / D t, D Q^{*} / D t\right)$. In the preheat zone, this term seems to drive the PDF towards the origin point. Finally, the right column of Fig. 8(a) shows that this term tends to drag back the joint PDF towards the Vieillefosse's tail in 

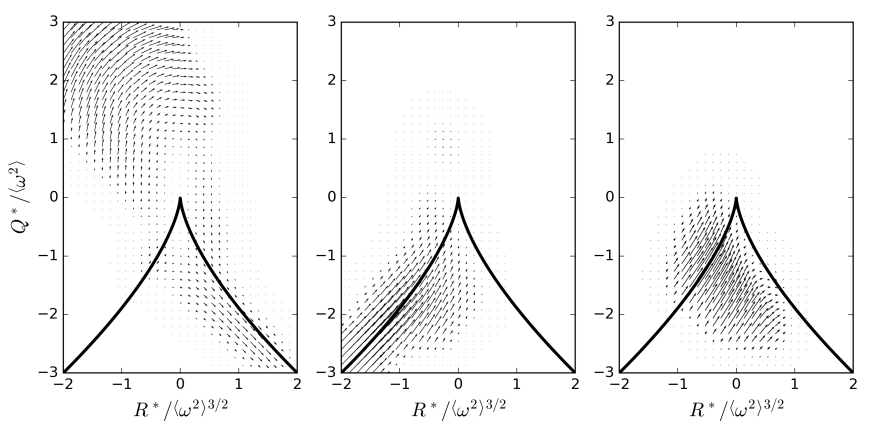

(a) $\left(\mathrm{I}_{Q^{*}}, \mathrm{I}_{R^{*}}\right)$
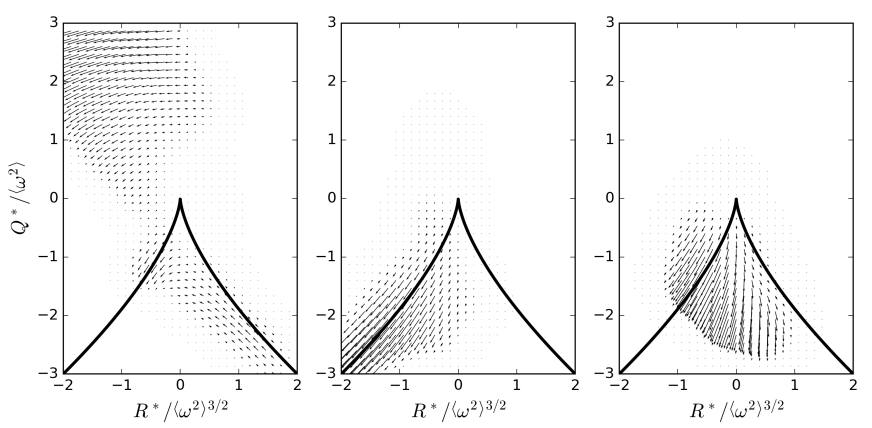

(b) $\left(\mathrm{II}_{Q^{*}}+\mathrm{III}_{Q^{*}}, \mathrm{II}_{R^{*}}+\mathrm{III}_{R^{*}}\right)$
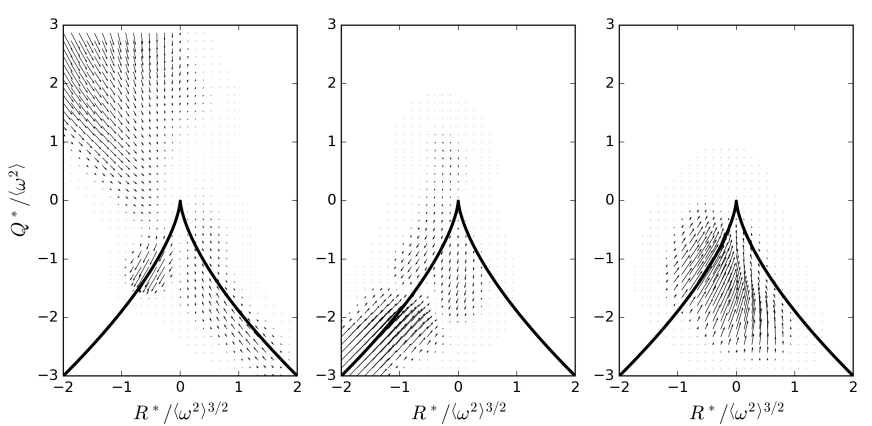

(c) $\left(\operatorname{IV}_{Q^{*}}, \operatorname{IV}_{R^{*}}\right)$

Figure 8. Contributions I (a), II+III (b), and IV (c) to the CMV as given by the LEE for $Q^{*}$ and $R^{*}$ (case F1) Left column: $c<0.01$ (fresh reactants), middle column: $0.01<c<0.50$, right column: $0.50<c<0.95$. 
the combustion zone. This is the main contribution that leads the tear-drop shape of the joint PDF to be recovered in the burned gases. From Fig. $8(\mathrm{~b})$, it is clear that the high probability of finding $\left(R^{*}, Q^{*}\right)$ on the left branch of the zero-discriminant line (i.e., $\left.\Delta^{*}=0.0\right)$ is mainly an outcome of the pressure terms, $\left(\mathrm{II}_{R^{*}}, \mathrm{II}_{Q^{*}}\right)$ and $\left(\mathrm{III}_{R^{*}}, \mathrm{III}_{Q^{*}}\right)$. In this low-Mach number reactive flow, the corresponding pressure effects are felt on the fresh reactants side and the left column of Fig. 8(b) puts into evidence the probability flux that drives the PDF from the Vieillefosse's tail, i.e., the right branch of the zero-discriminant line, towards its left counterpart. The probability flux along the left branch of the zero-discriminant line is significantly enhanced in the preheat zone, as it can be seen in the middle column of Fig. 8(b). However, the overall effect of the pressure terms becomes more distributed in the reacting zone (right column of Fig. 8(b)): it drives the PDF towards negative values of the second invariant $Q^{*}$ (i.e., strain-dominated flow). In the reacting zone, the increase in temperature makes the viscous contribution $\left(\mathrm{IV}_{R^{*}}, \mathrm{IV}_{Q^{*}}\right)$ significant (the value of the molecular viscosity is increased) and, as expected, the corresponding term tends to counteract the effects of the other terms so as to decrease the velocity gradient and drives the joint PDF towards the origin, see the right column of Fig. 8(c). This is however in sharp contrast with the behaviour of this term on the fresh reactants side (left column of Fig. 8(c)) and in the preheat zone (middle column of Fig. 8(c)). Indeed, in the fresh reactants region, this term induces a probability flux toward the left branch of the zero-discriminant line, which is rather similar to the one resulting from the pressure effects. In this region, the viscous contribution also generates a significant probability flux from the SF/S topology towards the origin. In the preheat zone (middle column of Fig. 8(c)), the probability fluxes that are induced by the viscous contribution $\left(\mathrm{IV}_{R^{*}}, \mathrm{IV}_{Q^{*}}\right)$ tend to maintain a high probability level for the $\mathrm{SN} / \mathrm{S} / \mathrm{S}$ topology in the vicinity of the left branch of the zero-discriminant line.

There exist some differences between the two contributions of the pressure fields namely the one of the pressure Hessian term $\left(\mathrm{II}_{R^{*}}, \mathrm{II}_{Q^{*}}\right)$ and the one of the baroclinic term $\left(\mathrm{III}_{R^{*}}, \mathrm{III}_{Q^{*}}\right)$. These differences are negligible in the reaction zone (right column of Fig. 9) but quite remarkable in the preheat zone (middle column of Fig. 9). Indeed, in the corresponding region, the probability flux generated by the baroclinic terms $\left(\mathrm{III}_{R^{*}}, \mathrm{III}_{Q^{*}}\right)$ seems to feature a direction that is opposite to the one associated to the pressure Hessian term $\left(\mathrm{II}_{R^{*}}, \mathrm{II}_{Q^{*}}\right)$. In fact, in the preheat region, the density variations are non-negligible, and the influence of the correlations between density, pressure and velocity gradients may differ between the two contributions. However, as it is confirmed by considering the sum of both $\left(\mathrm{II}_{R^{*}}, \mathrm{II}_{Q^{*}}\right)$ and $\left(\mathrm{III}_{R^{*}}, \mathrm{III}_{Q^{*}}\right)$, baroclinic effects remains rather moderate 

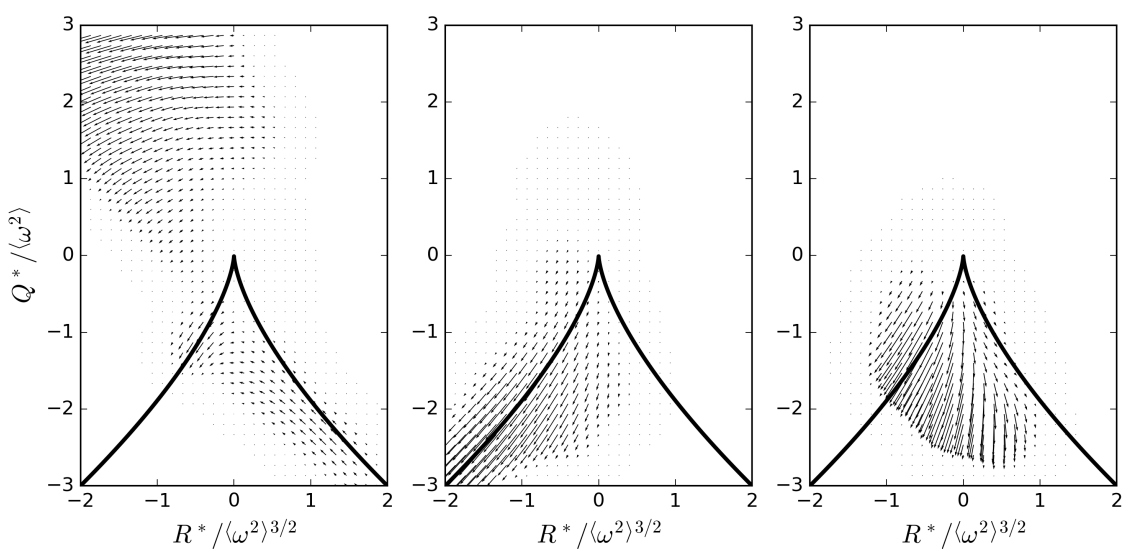

(a) $\left(\mathrm{II}_{Q^{*}}, \mathrm{II}_{R^{*}}\right)$
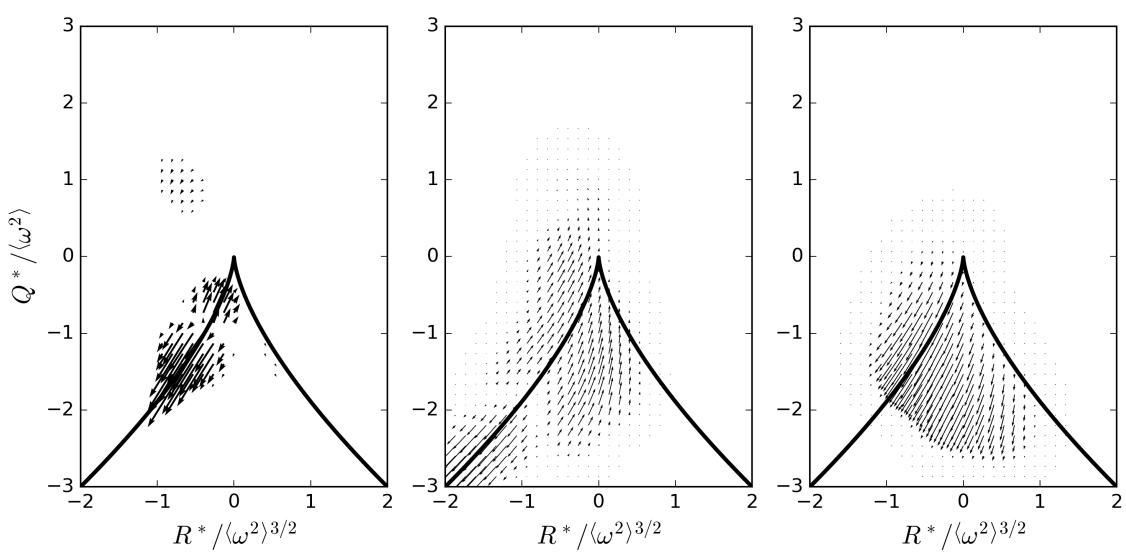

(b) $\left(\mathrm{III}_{Q^{*}}, \mathrm{III}_{R^{*}}\right)$

Figure 9. Contributions of the pressure Hessian term II (a) and baroclinic term III (b) to the CMV in the $\left(R^{*}, Q^{*}\right)$ plane (case F1). Left column: $c<0.01$ (fresh reactants), middle column: $0.01<c<0.50$, right column: $0.50<c<0.95$.

compared to the influence of the pressure Hessian terms in this weekly turbulent flame configuration. This corroborates some theoretical analyses, which previously emphasized the important role of the pressure Hessian and its influence on the topology of both scalar and velocity fields 

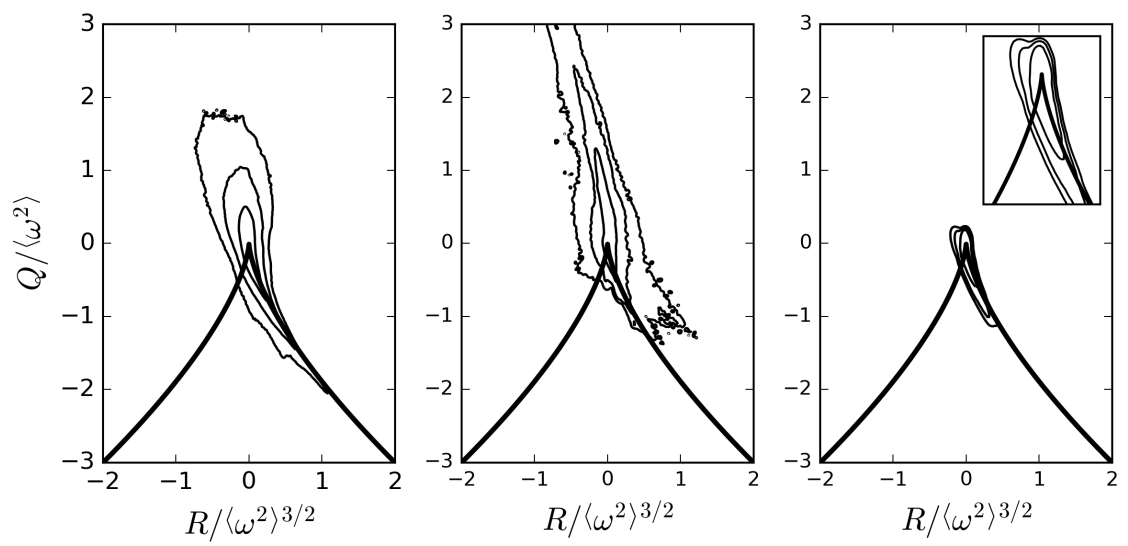

(a) Case F1
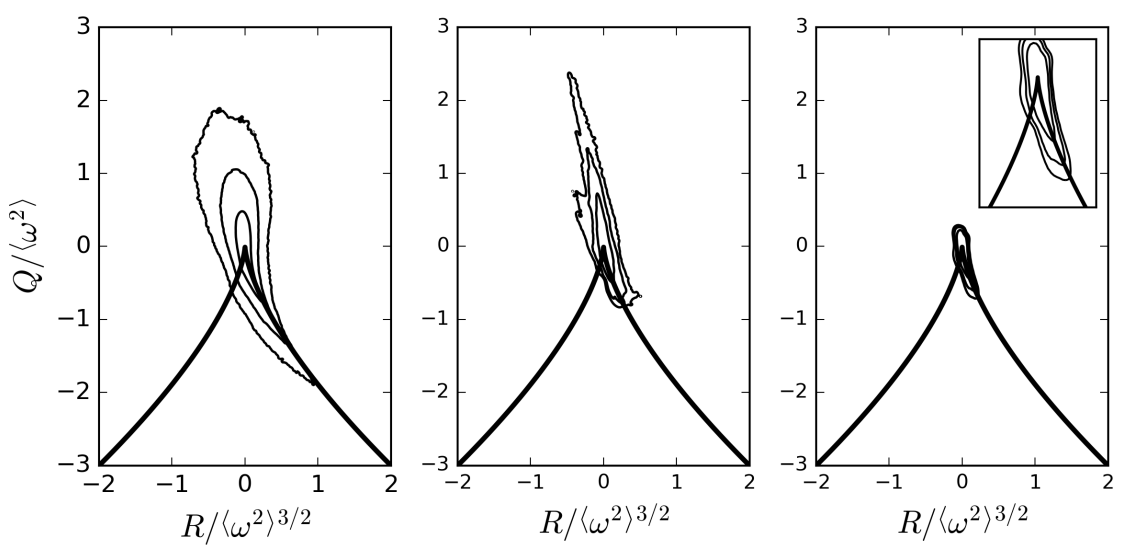

(b) Case F2

Figure 10. Iso-contours of the joint PDF of the third and second invariants $(R, Q)$ of the VGT in (a) case F1 and (b) case F2. Left column: fresh reactants $(c<0.01)$, middle: flame region $(0.01<c<0.95)$, right: burnt gases $(c>0.95)$. The three thin lines are the iso-contours of $\log _{10}$ (JPDF) equals to $-2.0,-1.0$, and 0.0 .

in turbulent flows featuring density variations ${ }^{49}$ and exothermal chemical reactions ${ }^{68}$. Finally, it is noteworthy that, on the fresh reactants side (left column of Fig. 9), the action of the baroclinic term is restricted to the vicinity of the left branch of the line $\Delta^{*}=0.0$. 
The results issued from the budget analysis reported above, which has been performed for the various terms present in the transport of $Q^{*}$ and $R^{*}$ seems to be quite consistent. Indeed, for expanding turbulent flame kernels such as those considered herein, the flow topology in the fresh reactants is slightly altered by pressure terms through the development of the kernel of combustion products. The preheat zone is significantly influenced by the expanding flame front, while the high levels of viscosity in the hot burning gases enhance the dissipation of velocity fluctuations and associated velocity gradients, which finally leads to the recovery of a HIT-like behavior within the kernel of hot burned products.
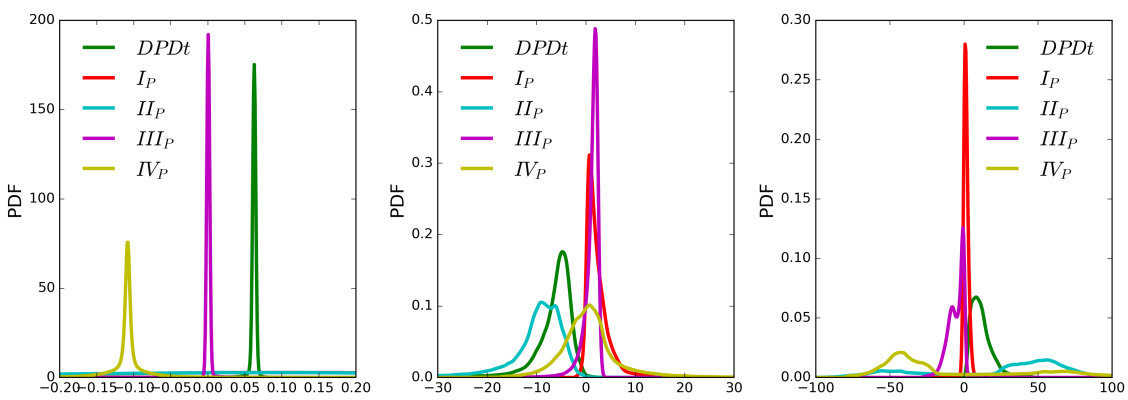

Figure 11. PDF of the various terms present in the LEE for the first invariant $P$ of the VGT (case F1). Left column: $c<0.01$ (fresh reactants), middle column: $0.01<c<0.50$, right column: $0.50<c<0.95$.

The evolution of the flow topology in these turbulent premixed reactive flows is further assessed through the consideration of the second and third invariants of the VGT $A$, i.e., $Q$ and $R$, which are now briefly analyzed. The joint PDF of the corresponding invariants is displayed in Fig. 10. It seems worth reminding that the first invariant $P$ of the VGT differs from zero and, in a first step of this analysis, its evolution is examined by considering the PDF of the various contributions that appear in its LEE, see Fig. 11. In the left column, it can be seen that the fresh reactants, which are slightly pushed by the expanding kernel, display a small positive value of the first invariant $P$. However, in the preheat zone, the flow is more significantly altered by the expanding flame kernel through the pressure Hessian term $\mathrm{II}_{P}$, leading to a large negative value of $D P / D t$ (the dilatation rate is increasing, i.e., $D \theta / D t$ is positive). In the reaction zone, positive values of the same contribution $\mathrm{II}_{P}$ tends to favor a return-to-zero of the first invariant $P$, which leads to recover the behavior of an almost incompressible HIT in the burnt products. 
The CMV associated to the invariants $Q$ and $R$ of the VGT $A$ are displayed in Fig. 12. In the unburnt reactants, the vector field is similar to the one relevant to standard HIT: the interaction between the invariants, i.e., contribution $\left(\mathrm{I}_{R}, \mathrm{I}_{Q}\right)$, drags the joint PDF up in the left half of the $(R, Q)$ plane and down in its right half associated to positive values of $R$. The pressure terms, i.e.,
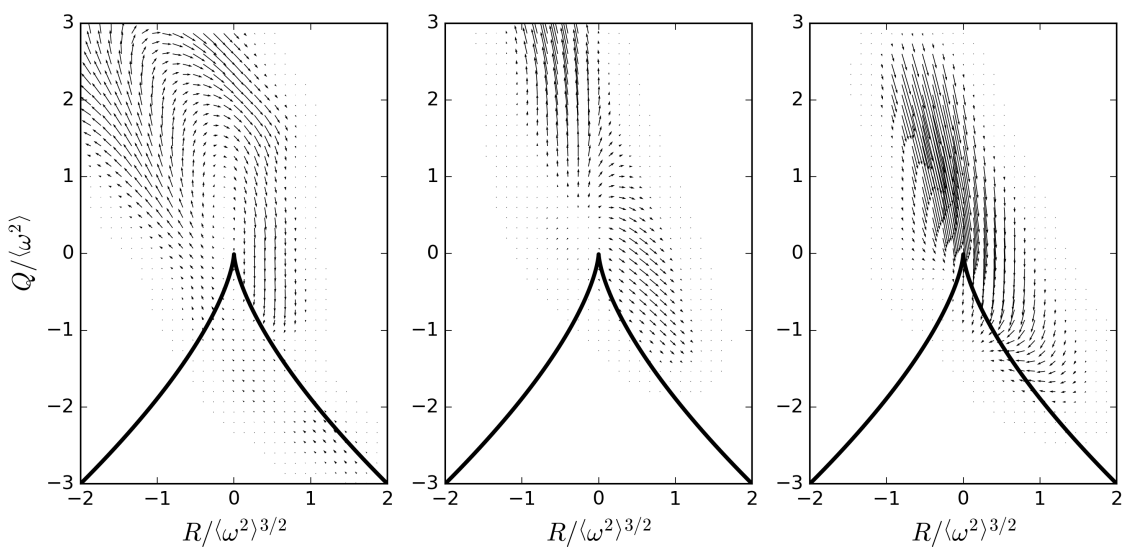

(a) Case F1
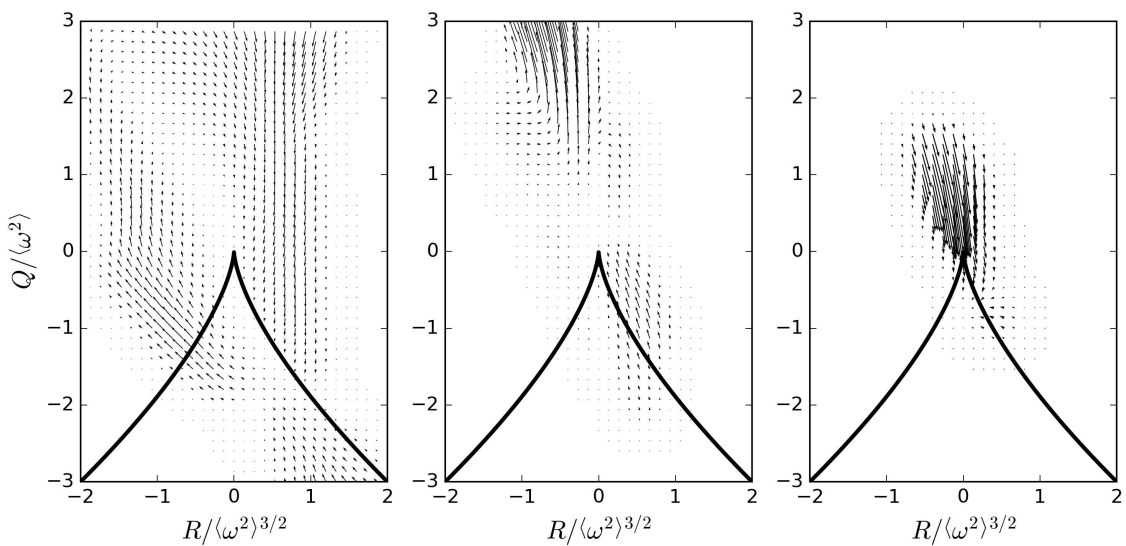

(b) Case F2

Figure 12. Conditional mean vectors in the $(R, Q)$ invariants plane for case F1 (a) and case F2 (b). Left column: $c<0.01$ (fresh reactants), middle column: $0.01<c<0.50$, right column: $0.50<c<0.95$. 

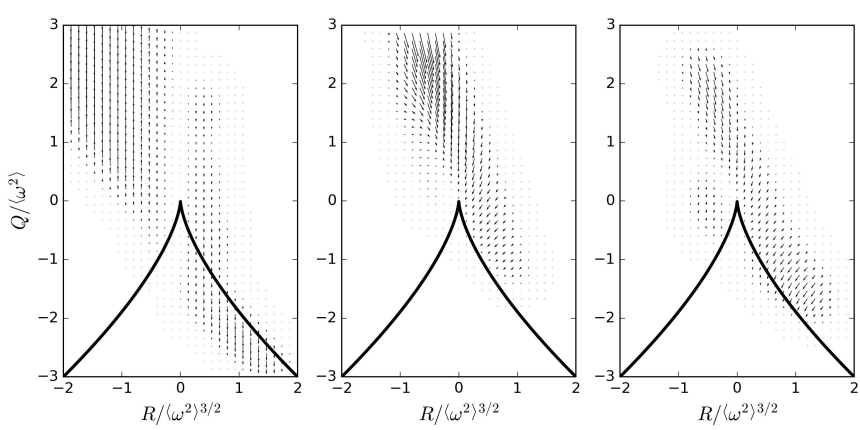

(a) $\left(\mathrm{I}_{Q}, \mathrm{I}_{R}\right)$
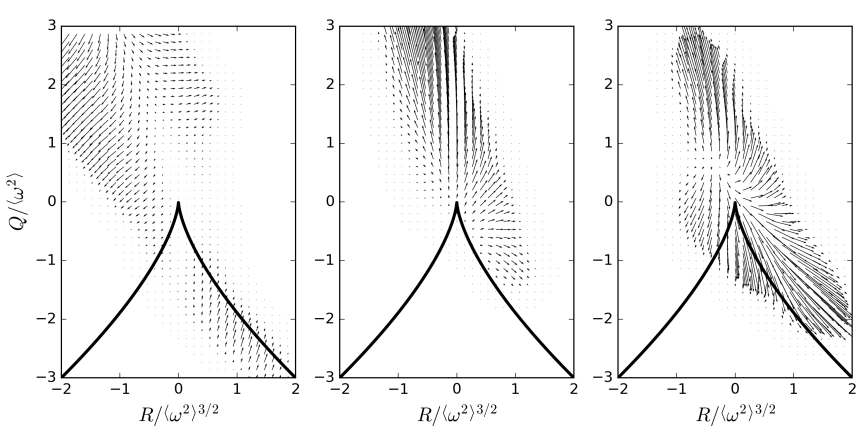

(b) $\left(\mathrm{II}_{Q}+\mathrm{III}_{Q}, \mathrm{II}_{R}+\mathrm{III}_{R}\right)$

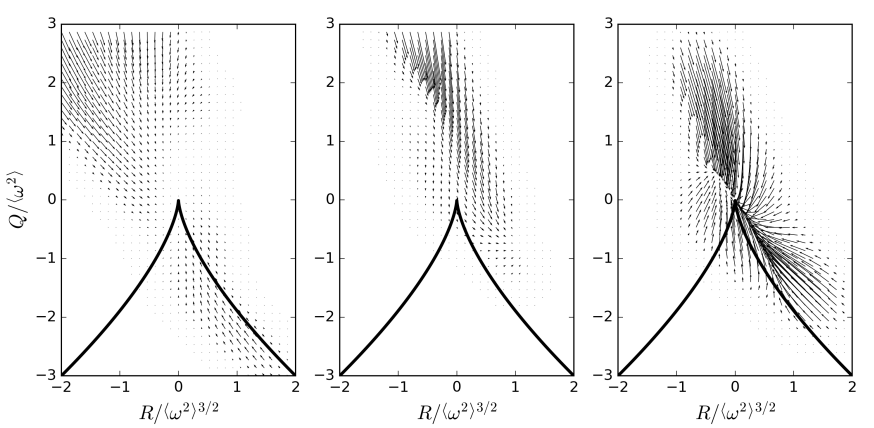

(c) $\left(\mathrm{IV}_{Q}, \mathrm{IV}_{R}\right)$

Figure 13. Contributions I (a), II+III (b), and IV (c) to the CMV as given by the LEE for $Q$ and $R$ (case F1). Left column: $c<0.01$ (fresh reactants), middle column: $0.01<c<0.50$, right column: $0.50<c<0.95$. 

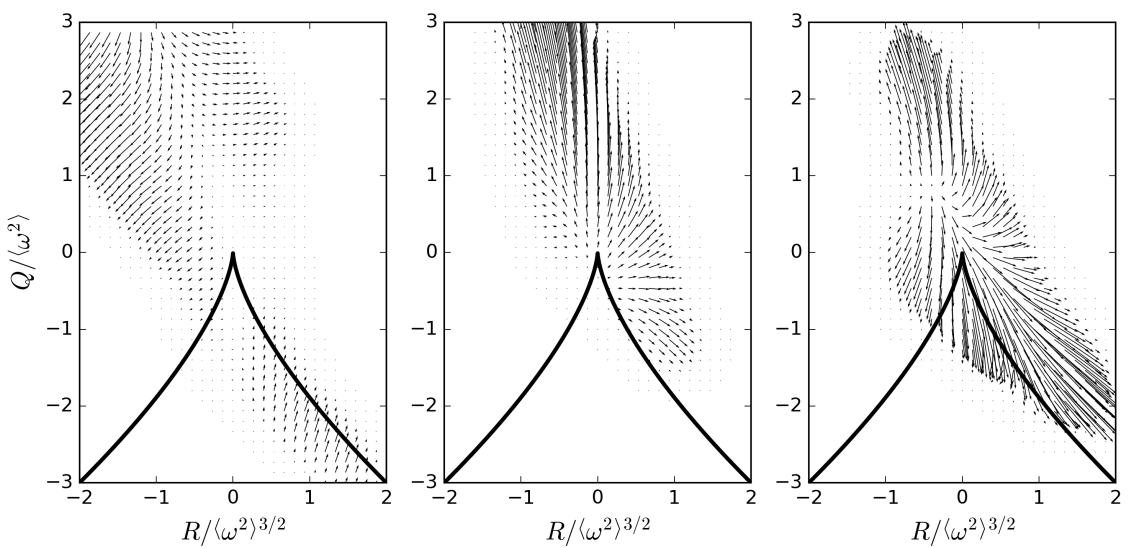

(a) $\left(\mathrm{II}_{Q}, \mathrm{II}_{R}\right)$
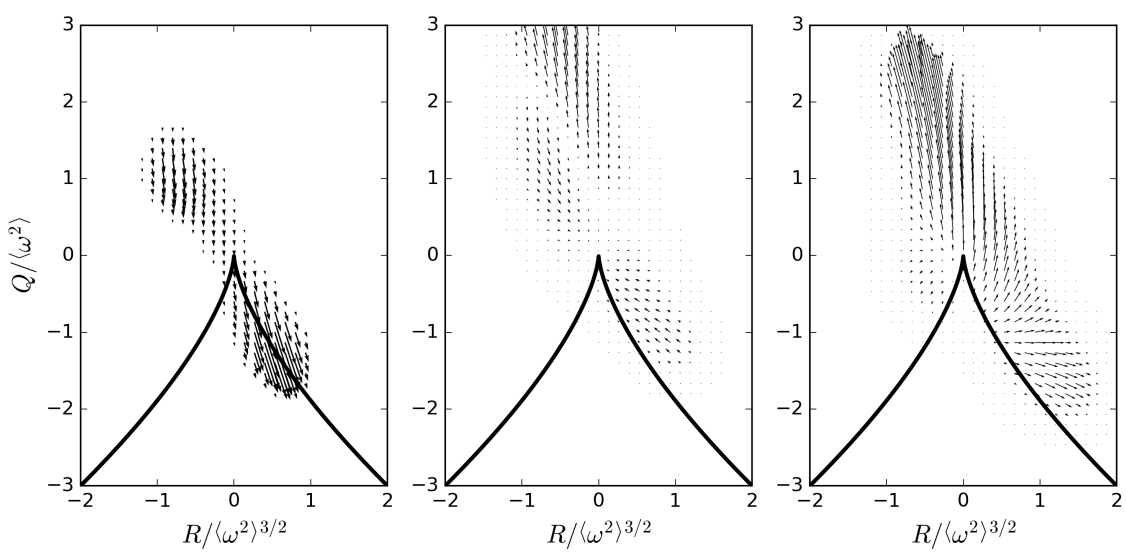

(b) $\left(\mathrm{III}_{Q}, \mathrm{III}_{R}\right)$

Figure 14. Contributions of the pressure Hessian term (a) and baroclinic term (b) to the CMV in the $(R, Q)$ plane (case F1). Left column: $c<0.01$ (fresh reactants), middle column: $0.01<c<0.50$, right column: $0.50<c<0.95$.

$\left(\mathrm{II}_{R}, \mathrm{II}_{Q}\right)$ and $\left(\mathrm{III}_{R}, \mathrm{III}_{Q}\right)$, tend to expand the joint PDF, while the viscous term $\left(\mathrm{IV}_{R}, \mathrm{IV}_{Q}\right)$ tends to bring it back to the origin point. The overall effect that results from all these contributions leads to a clockwise circulation $(D R / D t, D Q / D t)$ that maintains the tear-drop shape, as shown in the 
left column of Fig. 12(a). However, in the vicinity of the flame, the observed behaviour is quite different, especially for the interaction term between invariants $\left(\mathrm{I}_{R}, \mathrm{I}_{Q}\right)$. Indeed, in these regions, i.e., in the preheat and reaction regions associated to $0.01<c<0.95$, the vector $\left(\mathrm{I}_{R}, \mathrm{I}_{Q}\right)$ is no longer straightening the joint probability field up and down but instead it is pointing towards the origin point, as shown in Fig. 13. This is in contrast to the pressure terms, which tend to expand the joint PDF in both the preheat and the reacting regions, with a more significant contribution in the reacting region, as shown in Fig. 13(b). At the same time, the viscous term brings the joint probability towards the origin point, as it does on the fresh reactants side, see Fig. 13(c). In regard to the pressure terms, Fig. 14 shows that, in the preheat zone, the pressure Hessian term has a different sign compared to the baroclinic term, a behaviour similar to the one previously observed for the invariants $Q^{*}$ and $R^{*}$ of the traceless VGT.

To conclude, it should be emphasized that the same analysis has been conducted on flame F2. The obtained results are rather similar to those detailed above for flame F1. However, the turbulence dynamics tends to counteract - at least partly - the thermal expansion effects that are induced by the chemical reactions and resulting heat release. The corresponding results are not reported in this section just for the sake of conciseness and are instead briefly summarized in Appendix C.

\section{CONCLUSIONS}

Flow topology in weakly turbulent premixed flames is studied on the basis of velocity gradient tensor (VGT) invariants. Whatever the frameworks (geometrical and vectorial, streamline topology classification, spectral, structure function, etc.) retained to proceed with analyses directed towards a better understanding of turbulence, the velocity gradient plays a crucial role in one way or another. Thus, the velocity gradient is also central to turbulent combustion: the heat released by the flame leads to flow acceleration (this is a velocity gradient) and the velocity gradient associated to turbulent motion leads to strain and curvature of the flame front (variations of the flame surface area). The joint statistics of the VGT invariants in turbulent flames is analyzed using two DNS databases of flame kernel growth in initially homogeneous isotropic turbulence (HIT). The corresponding datasets correspond to turbulent flames that lie in the flamelet regime of turbulent combustion and are characterized by two distinct values of the Bray number. The imprints of the thermal expansion resulting from combustion exothermicity is put into evidence 
on the joint statistics of the second and third invariants (i.e., $Q^{*}$ and $R^{*}$ ) of the traceless VGT. The flow acceleration that takes place across local flamelets leads clearly to a significant increase in probability along the left branch of the zero discriminant curve $\left(\Delta^{*}=27 / 4 R^{* 2}+Q^{* 3}=0.0\right)$, especially for the case featuring the largest value of the Bray number (i.e., flame F1). The analysis of the present set of turbulent premixed flames databases shows that non-focal topologies are favoured compared to more standard conditions relevant to non-reactive incompressible turbulence. However, the origins of this specific behaviour remains unknown. Therefore, analyzing the rate of change of the VGT characteristics appears as a relevant way to get a better understanding of the behavior observed in turbulent flames. The origin of the corresponding probability fluxes is analyzed in details by considering the Lagrangian evolution equations (LEE) of the VGT invariants. Each term appearing in the RHS of the corresponding set of LEE is scrutinized and the corresponding effects are pictured as normalized conditional mean vectors in the $\left(R^{*}, Q^{*}\right)$ coordinates. The obtained results show that the pressure Hessian contribution is mostly responsible for the observed behavior, a conclusion that corroborates recent theoretical analyses which emphasized the important role of this contribution and its influence on the topology of both scalar and velocity fields in turbulent reactive flows. In this respect, a recent study ${ }^{52}$ devoted to the evolution of the reactive scalar gradient orientation in the strain-rate tensor eigenframe clearly puts into evidence a significant rotation of the strain-rate tensor principal axes that can exceed ninety degrees as it evolves towards the premixed flame front. The consideration of the reactive scalar gradient orientation vector showed that the corresponding rotation mechanism does involve pressure variations through the pressure Hessian, which was therefore thought to be responsible for the observed behavior. The present set of computational results brings additional support to this conclusion. It also emphasizes the influence of non-local or indirect thermal expansion effects ${ }^{31}$, which cannot be represented by local flamelet crossing only and must take into account the flame wrinkling effects. Finally, the present work devoted to the first analysis of LEE for the VGT invariants in turbulent premixed flames certainly does not give definitive answers to all questions regarding the evolution of the corresponding joint-statistics but, at least, the corresponding set of original results leads to new insights and it provides a solid basis for further analyses. 


\section{ACKNOWLEDGMENTS}

The authors are indebted to Marcos Onofre Ramos for technical assistance.

\section{Appendix A: LEE for the invariants of the traceless part of the VGT tensor}

From Eqs. (15a)-(15c), it is possible to deduce the transport equations for the invariants of the deviatoric, i.e., traceless, VGT $\boldsymbol{A}^{*}$. Indeed, as the tensor $\boldsymbol{A}^{*} \boldsymbol{A}^{*}$ and $\boldsymbol{A}^{*} \boldsymbol{A}^{*} \boldsymbol{A}^{*}$ can be written as follows:

$$
\begin{aligned}
\left(A^{*} A^{*}\right)_{i j} & =A_{i k}^{*} A_{k j}^{*}=\left(A_{i k}+\frac{\delta_{i k}}{3} P\right)\left(A_{k j}+\frac{\delta_{k j}}{3} P\right) \\
& =(A A)_{i j}+\frac{2 P}{3} A_{i j}+\frac{1}{9} \delta_{i j} P^{2} \\
\left(A^{*} A^{*} A^{*}\right)_{i j} & =\left(A^{*} A^{*}\right)_{i k} A_{k j}^{*}=\left((A A)_{i k}+\frac{2 P}{3} A_{i k}+\frac{1}{9} \delta_{i k} P^{2}\right)\left(A_{k j}+\frac{\delta_{k j}}{3} P\right) \\
& =(A A A)_{i j}+\frac{P}{3}(A A)_{i j}+\frac{2 P}{3}(A A)_{i j}+\frac{2 P^{2}}{9} A_{i j}+\frac{P^{3}}{9}+\frac{P^{2}}{9} A_{i j}+\frac{1}{27} \delta_{i j} P^{3} \\
& =(A A A)_{i j}+P(A A)_{i j}+\frac{P^{2}}{3} A_{i j}+\frac{1}{27} \delta_{i j} P^{3},
\end{aligned}
$$

the invariants of $\boldsymbol{A}^{*}$ can be expressed using $P, Q$, and $R$,

$$
\begin{aligned}
Q^{*} & =-\frac{1}{2}\left(A^{*} A^{*}\right)_{i i}=-\frac{1}{2}\left[(A A)_{i i}+\frac{2 P}{3} A_{i i}+\frac{1}{3} P^{2}\right] \\
& =-\frac{1}{2}\left[(A A)_{i i}-\frac{P^{2}}{3}\right]=-\frac{1}{2}\left[P^{2}-2 Q-\frac{P^{2}}{3}\right] \\
& =Q-\frac{1}{3} P^{2} \\
R^{*} & =-\frac{1}{3}\left(A^{*} A^{*} A^{*}\right)_{i i}=-\frac{1}{3}\left[(A A A)_{i i}+P(A A)_{i i}+\frac{P^{2}}{3} A_{i i}+\frac{1}{9} P^{3}\right] \\
& =-\frac{1}{3}\left[(A A A)_{i i}+P(A A)_{i i}-\frac{P^{3}}{3}+\frac{1}{9} P^{3}\right] \\
& =-\frac{1}{3}\left[\left(3 P Q-P^{3}-3 R\right)+P\left(P^{2}-2 Q\right)-\frac{2 P^{3}}{9}\right] \\
& =R-\frac{1}{3} P Q+\frac{2}{27} P^{3}
\end{aligned}
$$


Applying the chain-rule of differential operations to the above equations leads to the transport equations for $P^{*}, Q^{*}$, and $R^{*}$ that can be written as

$$
\begin{aligned}
\frac{D P^{*}}{D t} & =0 \\
\frac{D Q^{*}}{D t} & =\frac{D Q}{D t}-\frac{2 P}{3} \frac{D P}{D t} \\
& =\mathrm{I}_{Q^{*}}+\mathrm{II}_{Q^{*}}+\mathrm{III}_{Q^{*}}+\mathrm{IV}_{Q^{*}} \\
\frac{D R^{*}}{D t} & =\frac{D R}{D t}-\frac{1}{3}\left(P \frac{D Q}{D t}+Q \frac{D P}{D t}\right)+\frac{2 P^{2}}{9} \frac{D P}{D t} \\
& =\mathrm{I}_{R^{*}}+\mathrm{II}_{R^{*}}+\mathrm{III}_{R^{*}}+\mathrm{IV}_{R^{*}}
\end{aligned}
$$

where the various terms present in the RHS are given by

$$
\begin{aligned}
\mathrm{I}_{Q^{*}} & =\mathrm{I}_{Q}-\frac{2 P}{3} \mathrm{I}_{P}=-\frac{2}{3} P^{3}+\frac{7}{3} P Q-3 R \\
\mathrm{II}_{Q^{*}} & =\frac{1}{3} P \mathscr{H}_{i i}^{p}+A_{i j} \mathscr{H}_{j i}^{p} \\
\mathrm{III}_{Q^{*}} & =\frac{1}{3} P \mathscr{H}_{i i}^{b}+A_{i j} \mathscr{H}_{j i}^{b} \\
\mathrm{IV}_{Q^{*}} & =-\frac{1}{3} P \mathscr{T}_{i i}-A_{i j} \mathscr{T}_{j i}
\end{aligned}
$$

and

$$
\begin{aligned}
\mathrm{I}_{R^{*}} & =\mathrm{I}_{R}-\frac{1}{3}\left(P \mathrm{I}_{Q}+Q \mathrm{I}_{P}\right)+\frac{2 P^{2}}{9} \mathrm{I}_{P}=\frac{2}{9} P^{4}-\frac{10}{9} P^{2} Q+\frac{2}{3} Q^{2}+2 P R \\
\mathrm{II}_{R^{*}} & =\frac{6 Q-P^{2}}{9} \mathscr{H}_{i i}^{p}+\frac{2 P}{3} A_{i j} \mathscr{H}_{j i}^{p}+A_{i k} A_{k j} \mathscr{H}_{k i}^{p} \\
\mathrm{III}_{R^{*}} & =\frac{6 Q-P^{2}}{9} \mathscr{H}_{i i}^{b}+\frac{2 P}{3} A_{i j} \mathscr{H}_{j i}^{b}+A_{i k} A_{k j} \mathscr{H}_{k i}^{b} \\
\mathrm{IV}_{R^{*}} & =-\frac{6 Q-P^{2}}{9} \mathscr{T}_{i i}-\frac{2 P}{3} A_{i j} \mathscr{T}_{j i}-A_{i k} A_{k j} \mathscr{T}_{k i}
\end{aligned}
$$

Appendix B: Second and third invariants of the traceless VGT in one-dimensional laminar premixed flames

Let us consider a steady one-dimensional planar unstrained laminar premixed flame that just for the sake of simplificity - is assumed to propagate along the $x_{1}$-direction. In this case, the velocity gradient tensor can be written as follows: 


$$
\boldsymbol{A}=\left[\begin{array}{ccc}
E_{f} & 0 & 0 \\
0 & 0 & 0 \\
0 & 0 & 0
\end{array}\right]
$$

where $E_{f}$ is a positive-defined parameter, which is related to the thermal expansion. Assuming a linear approximation of the laminar flame profile, it is $E_{f}=\tau S_{L}^{0} / \delta_{L}^{0}$.

The traceless tensor $\boldsymbol{A}^{*}$ can be readily deduced from the above expression of $\boldsymbol{A}$

$$
\boldsymbol{A}^{*}=\left[\begin{array}{ccc}
\xi_{1} & 0 & 0 \\
0 & \xi_{2} & 0 \\
0 & 0 & \xi_{3}
\end{array}\right]=\left[\begin{array}{ccc}
2 E_{f} / 3 & 0 & 0 \\
0 & -E_{f} / 3 & 0 \\
0 & 0 & -E_{f} / 3
\end{array}\right]
$$

where $\xi_{i}$ with $i=1, \ldots, 3$ denotes the three eigenvalues of $\boldsymbol{A}^{*}$. Since the tensor is traceless, it is easy to show that its invariants are given by the following expressions: $P^{*}=-\left(\xi_{1}+\xi_{2}+\xi_{3}\right)=$ $0.0, Q^{*}=\xi_{1} \xi_{2}+\xi_{2} \xi_{3}+\xi_{1} \xi_{3}=-E_{f}^{2} / 3$, and $R^{*}=-\xi_{1} \xi_{2} \xi_{3}=-2 E_{f}^{3} / 27$. It is remarkable that these values correspond to zero values of the discriminant $\Delta^{*}$ of the characteristic polynomial of $\boldsymbol{A}^{*}$ since they verify $4 Q^{* 3}-27 R^{* 2}=0.0$. Therefore, in the the map of the invariants $\left(R^{*}, Q^{*}\right)$, the corresponding solutions are located on the curve $\Delta^{*}=0.0$. Moreover, since $R^{*}=-2 E_{f}^{3} / 27<0$, the couples $\left(R^{*}, Q^{*}\right)$ associated to the reference laminar premixed flame are located on the left branch of $\Delta^{*}=0.0$.

\section{Appendix C: LEE for the invariants of the VGT in case F2}

The invariants of the VGT $\boldsymbol{A}$ as well as those of its traceless counterparts $\boldsymbol{A}^{*}$ have been also scrutinized for case F2. The obtained results are rather similar to those obtained for case F1. There are, however, some differences which confirm that, as expected, the impact of the thermal expansion is less significant compared to case F1. In this respect, Fig. 7(b) shows that the JPDF is not that biased towards the left branch of $\Delta^{*}=0.0$ in the flame zone compared to the JPDF obtained for the flame F1. Accordingly, the analysis of the various terms that appear in the LEE of $Q^{*}$ and $R^{*}$ confirms that the terms which lead to the corresponding probability fluxes are much smaller in case F2, see Figs. 8 and 15. Similar conclusions can be drawn from the analysis of the LEE of the invariants of $\boldsymbol{A}$ that are not reported herein just for the sake of conciseness. In any case, although the impact of the flame is less significant in case F2, the way it modifies the 

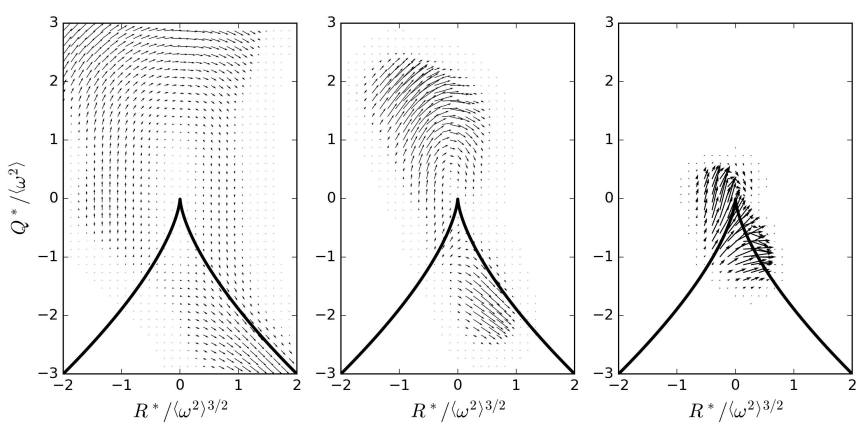

(a) $\left(\mathrm{I}_{Q^{*}}, \mathrm{I}_{R^{*}}\right)$
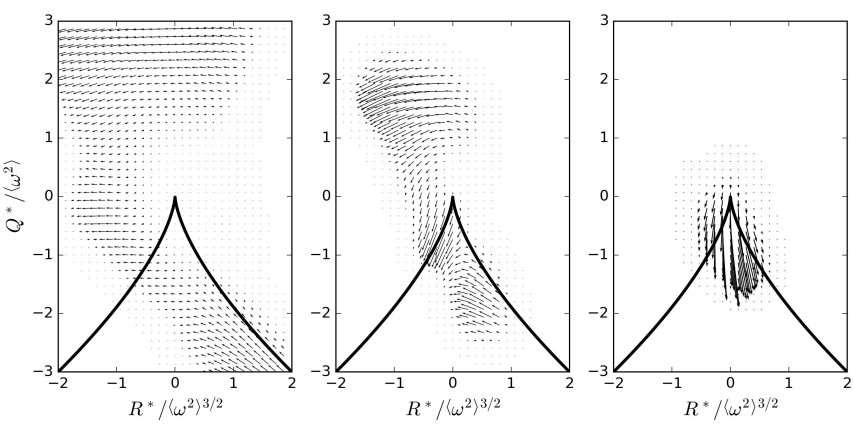

(b) $\left(\mathrm{II}_{Q^{*}}+\mathrm{III}_{Q^{*}}, \mathrm{II}_{R^{*}}+\mathrm{III}_{R^{*}}\right)$
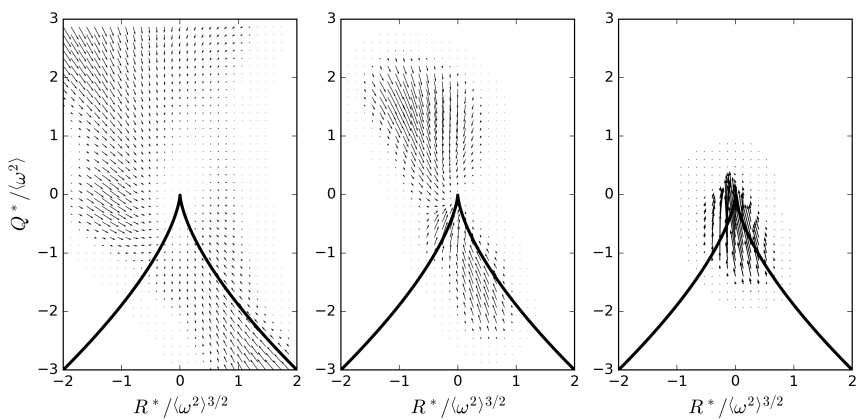

(c) $\left(\mathrm{IV}_{Q^{*}}, \mathrm{IV}_{R^{*}}\right)$

Figure 15. Contributions I (a), II+III (b), and IV (c) to the CMV as given by the LEE for $Q^{*}$ and $R^{*}$ (case F2) Left column: $c<0.01$ (fresh reactants), middle column: $0.01<c<0.50$, right column: $0.50<c<0.95$. 
invariants of the VGT and traceless VGT remains the same as the one it modifies them in case F1.

\section{DATA AVAILABILITY STATEMENT}

The data that support the findings of this study are available from the corresponding author upon reasonable request.

\section{REFERENCES}

${ }^{1}$ C. Dopazo and E. E. O’Brien, “An approach to the autoignition of a turbulent mixture," Acta Astronautica 1, 1239-1266 (1974).

${ }^{2}$ Y. Y. Kuo and E. E. O’Brien, “Two-point probability density function closure applied to a diffusive-reactive system," The Physics of Fluids 24, 194-201 (1981).

${ }^{3}$ R. E. Meyers and E. E. O'Brien, "The joint PDF of a scalar and its gradient at a point in a turbulent fluid," Combustion Science and Technology 26, 123-134 (1981).

${ }^{4} \mathrm{M}$. Z. Wu and E. E. O'Brien, "Prediction of single-point temperature statistics in a half-heated grid flow," Combustion Science and Technology 29, 53-66 (1982).

${ }^{5}$ T. H. J. Hsieh and E. E. O’Brien, "Prediction of single point concentration statistics in a chemically-reactive, turbulent, grid flow," Combustion Science and Technology 46, 267-287 (1986).

${ }^{6}$ V. Eswaran and E. E. O'Brien, "Simulations of scalar mixing in grid turbulence using an eddydamped closure model," Physics of Fluids A: Fluid Dynamics 1, 537-548 (1989).

${ }^{7}$ T. L. Jiang and E. E. O’Brien, "Simulation of scalar mixing by stationary isotropic turbulence," Physics of Fluids A: Fluid Dynamics 3, 1612-1624 (1991).

${ }^{8}$ E. E. O’Brien and T. L. Jiang, "The conditional dissipation rate of an initially binary scalar in homogeneous turbulence," Physics of Fluids A: Fluid Dynamics 3, 3121-3123 (1991).

${ }^{9}$ F. Gao and E. E. O’Brien, "Direct numerical simulations of reacting flows in homogeneous turbulence," AIChE Journal 37, 1459-1470 (1991).

${ }^{10}$ E. E. O’Brien, "Spectra of single reactants in homogeneous turbulence," in Frontiers in fluid mechanics: A collection of research papers written in commemoration of the 65th birthday of Stanley Corrsin (Springer-Verlag, 1985) pp. 113-122. 
${ }^{11}$ F. Gao and E. E. O’Brien, “A large-eddy simulation scheme for turbulent reacting flows," Physics of Fluids A: Fluid Dynamics 5, 1282-1284 (1993).

${ }^{12}$ S. B. Pope, "PDF methods for turbulent reactive flows," Progress in Energy and Combustion Science 11, 119-192 (1985).

${ }^{13}$ R. Borghi, “Turbulent combustion modelling," Progress in Energy and Combustion Science 14, 245-292 (1988).

${ }^{14}$ C. Dopazo, "Recent developments in PDF methods," in Turbulent Reacting Flows (Academic Press, 1994) pp. 375-474.

${ }^{15} \mathrm{P}$. Givi, "Filtered density function for subgrid scale modeling of turbulent combustion," AIAA Journal 44, 16-23 (2006).

${ }^{16}$ A. N. Lipatnikov and J. Chomiak, "Effects of premixed flames on turbulence and turbulent scalar transport," Progress in Energy and Combustion Science 36, 1-102 (2010).

${ }^{17}$ V. A. Sabelnikov and A. N. Lipatnikov, "Recent advances in understanding of thermal expansion effects in premixed turbulent flames," Annual Review of Fluid Mechanics 49, 91-117 (2017).

${ }^{18}$ T. Mantel and R. Borghi, “A new model of premixed wrinkled flame based on a scalar dissipation equation," Combustion and Flame 96, 443-457 (1994).

${ }^{19}$ A. Mura and R. Borghi, "Towards an extended scalar dissipation equation for turbulent premixed combustion," Combustion and Flame 133, 193-196 (2003).

${ }^{20}$ A. Mura, F. Galzin, and R. Borghi, “A unified PDF-flamelet model for turbulent premixed combustion," Combustion Science and Technology 175, 1573-1609 (2003).

${ }^{21}$ N. Swaminathan and K. N. C. Bray, "Effect of dilatation on scalar dissipation in turbulent premixed flames," Combustion and Flame 143, 569-565 (2005).

${ }^{22}$ N. Chakraborty and N. Swaminathan, "Influence of the Damköhler number on turbulencescalar interaction in premixed flames, Part I: physical insight," Physics of Fluids 19, 045103.1045103.10 (2007).

${ }^{23}$ A. Mura, K. Tsuboi, and T. Hasegawa, "Modelling of the correlation between velocity and reactive scalar gradients in turbulent premixed flames based on DNS data," Combustion Theory and Modelling 12, 671-698 (2008).

${ }^{24}$ A. Mura, V. Robin, M. Champion, and T. Hasegawa, "Small scale features of velocity and scalar fields in turbulent premixed flames," Flow Turbulence and Combustion 82, 339-358 (2009).

${ }^{25} \mathrm{~A}$. Mura and M. Champion, "Relevance of the bray number in the small-scale modeling of turbulent premixed flames," Combustion and Flame 156, 729-733 (2009). 
${ }^{26}$ N. Chakraborty, M. Champion, A. Mura, and N. Swaminathan, "Scalar dissipation rate approach," in Turbulent premixed flames, edited by N. Swaminathan and K. Bray (Cambridge University Press, 2011) pp. 74-102.

${ }^{27}$ L. Cifuentes, C. Dopazo, J. Martin, and C. Jimenez, "Local flow topologies and scalar structures in a turbulent premixed flame," Physics of Fluids 26, 065108 (2014).

${ }^{28}$ M. Danish, S. Suman, and S. S. Girimaji, "Influence of flow topology and dilatation on scalar mixing in compressible turbulence," Journal of Fluid Mechanics 793, 633-655 (2016).

${ }^{29}$ R. Boukharfane, Z. Bouali, and A. Mura, "Evolution of scalar and velocity dynamics in planar shock-turbulence interaction," Shock Waves 28, 1117-1141 (2018).

${ }^{30}$ Y. Tian, F. A. Jaberi, and D. Livescu, "Density effects on post-shock turbulence structure and dynamics," Journal of Fluid Mechanics 880, 935-968 (2019).

${ }^{31}$ V. Robin, A. Mura, and M. Champion, "Direct and indirect thermal expansion effects in turbulent premixed flames, Journal of Fluid Mechanics 689, 149-182 (2011).

${ }^{32}$ H. Kolla, E. R. Hawkes, A. R. Kerstein, N. Swaminathan, and J. H. Chen, "On velocity and reactive scalar spectra in turbulent premixed flames," Journal of Fluid Mechanics 754, 456-487 (2014).

${ }^{33}$ L. Cifuentes, C. Dopazo, J. Martin, P. Domingo, and L. Vervisch, "Effects of the local flow topologies upon the structure of a premixed methane-air turbulent jet flame," Flow Turbulence and Combustion 96, 535-546 (2016).

${ }^{34}$ D. Wacks, I. Konstantinou, and N. Chakraborty, "Effects of lewis number on the statistics of the invariants of the velocity gradient tensor and local flow topologies in turbulent premixed flames," Proceedings of the Royal Society A: Mathematical, Physical and Engineering Sciences 474, 20170706 (2018).

${ }^{35}$ N. Chakraborty, D. H. Wacks, S. Ketterl, M. Klein, and H. G. Im, "Scalar dissipation rate transport conditional on flow topologies in different regimes of premixed turbulent combustion," Proceedings of the Combustion Institute 37, 2353-2361 (2019).

${ }^{36}$ V. A. Sabelnikov, L. A. N., N. Nikitin, S. Nishiki, and T. Hasegawa, "Application of helmholtzhodge decomposition and conditioned structure functions to exploring influence of premixed combustion on turbulence upstream of the flame," Proceedings of the Combustion Institute (2020), https://doi.org/10.1016/j.proci.2020.09.015.

${ }^{37}$ D. H. Wacks, N. Chakraborty, M. Klein, P. G. Arias, and H. G. Im, "Flow topologies in different regimes of premixed turbulent combustion: a direct numerical simulation analysis," Physical 
Review Fluids 1, 083401 (2016).

${ }^{38}$ V. A. Sabelnikov, L. A. N., N. Nikitin, S. Nishiki, and T. Hasegawa, "Solenoidal and potential velocity fields in weakly turbulent premixed flames," Proceedings of the Combustion Institute (2020), https://doi.org/10.1016/j.proci.2020.09.016.

${ }^{39}$ K. N. C. Bray, P. A. Libby, G. J. Masuya, and J. B. Moss, “Turbulence production in premixed turbulent flames," Combustion Science and Technology 25, 127-140 (1981).

${ }^{40}$ P. A. Libby and K. N. C. Bray, “Counter gradient diffusion in premixed turbulent flames,” AIAA Journal 19, 205-213 (1981).

${ }^{41}$ P. Bailly, M. Champion, and D. Garreton, "Counter-gradient diffusion in a confined turbulent premixed flame," Physics of Fluids 9, 766-775 (1997).

${ }^{42}$ K. N. C. Bray, M. Champion, and P. A. Libby, "Premixed flames in stagnating turbulence: Part IV. A new theory for the Reynolds stresses and Reynolds fluxes applied to impinging flows," Combustion and Flame 120, 1-18 (2000).

${ }^{43}$ V. Robin, M. Champion, and A. Mura, “A second-order model for turbulent reactive flows with variable equivalence ratio," Combustion Science and Technology 180, 1709-1734 (2008).

${ }^{44}$ P. E. Hamlington, A. Y. Poludnenko, and E. S. Oran, "Interactions between turbulence and flames in premixed reacting flows," Physics of Fluids 23, 125111 (2011).

${ }^{45}$ M. S. Chong, A. E. Perry, and B. J. Cantwell, “A general classification of three-dimensional flow fields," Physics of Fluids A: Fluid Dynamics 2, 765-777 (1990).

${ }^{46}$ S. Pirozzoli and F. Grasso, "Direct numerical simulations of isotropic compressible turbulence: Influence of compressibility on dynamics and structures," Physics of Fluids 16, 4386-4407 (2004).

${ }^{47}$ Y. B. Chu and X. Y. Lu, “Topological evolution in compressible turbulent boundary layers," Journal of Fluid Mechanics 733, 414-438 (2013).

${ }^{48}$ J. Martin, A. Ooi, C. Dopazo, M. S. Chong, and J. Soria, "The inverse diffusion time scale of velocity gradients in homogeneous isotropic turbulence," Physics of Fluids 9, 814 (1997).

${ }^{49}$ M. Gonzalez and P. Paranthoën, "Effects of variable mass density on the kinematics of scalar gradient," Physics of Fluids 23, 075107 (2011).

${ }^{50} \mathrm{M}$. Gonzalez, “Analyzing the effect of dilatation on the velocity gradient tensor using a model problem," Springer Nature Applied Sciences 2, 1793 (2020).

${ }^{51}$ J. Ryu and D. Livescu, "Turbulence structure behind the shock in canonical shock-vortical turbulence interaction," Journal of Fluid Mechanics 756, R1-1-R1-12 (2014). 
${ }^{52}$ S. Zhao, A. Er-raiy, Z. Bouali, and A. Mura, "Dynamics and kinematics of the reactive scalar gradient in weakly turbulent premixed flames," Combustion and Flame 198, 436-454 (2018).

${ }^{53}$ J. Reveillon, C. Péra, and Z. Bouali, "Examples of the potential of DNS for the understanding of reactive multiphase flows," International Journal of Spray and Combustion Dynamics 3, 63-92 (2011).

${ }^{54} \mathrm{~F}$. A. Williams, "Criteria for existence of wrinkled laminar flame structure of turbulent premixed flames," Combustion and Flame 26, 269-270 (1976).

${ }^{55} \mathrm{R}$. Borghi, “On the structure and morphology of turbulent premixed flames," in Recent Advances in the Aerospace Science, edited by C. Casci (Plenum Publishing, 1985) pp. 117-138.

${ }^{56}$ N. Peters, "Laminar flamelet concepts in turbulent combustion," Proceedings of the Combustion Institute 21, 1231-1250 (1986).

${ }^{57}$ D. Veynante, A. Trouvé, K. N. C. Bray, and T. Mantel, "Gradient and counter-gradient scalar transport in turbulent premixed flames," Journal of Fluid Mechanics 332, 263-293 (1997).

${ }^{58} \mathrm{Y}$. Ju and K. Maruta, "Microscale combustion: Technology development and fundamental research," Progress in Energy and Combustion Science 37, 669-715 (2011).

${ }^{59}$ S. Tanaka, M. Shimura, N. Fukushima, M. Tanahashi, and T. Miyauchi, "DNS of turbulent swirling premixed flame in a micro gas turbine combustor," Proceedings of the Combustion Institute 33, 3293-3300 (2011).

${ }^{60} \mathrm{~T}$. Passot and A. Pouquet, "Numerical simulation of compressible homogeneous flows in the turbulent regime," Journal of Fluid Mechanics 181, 441-466 (1987).

${ }^{61}$ E. Samson, A. Mura, B. Renou, and M. Boukhalfa, "Characterization of the local flame structure and the flame surface density for freely-propagating premixed flames at various Lewis numbers," Combustion Science and Technology 174-179, 143 (2002).

${ }^{62}$ K. Kha, V. Robin, A. Mura, and M. Champion, "Implications of laminar flame finite thickness on the structure of turbulent premixed flames," Journal of Fluid Mechanics 787, 116-147 (2016).

${ }^{63}$ A. E. Perry and M. S. Chong, "A description of eddying motions and flow patterns using criticalpoint concepts," Annual Review of Fluid Mechanics 19, 125-155 (1987).

${ }^{64}$ A. E. Perry and M. S. Chong, "Topology of flow patterns in vortex motions and turbulence," Applied Scientific Research 53, 357-374 (1994).

${ }^{65} \mathrm{P}$. Vieillefosse, "Local interaction between vorticity and shear in a perfect incompressible fluid," Journal de Physique 43, 837-842 (1982). 
${ }^{66}$ J. Martin, A. Ooi, M. S. Chong, and J. Soria, "Dynamics of the velocity gradient tensor invariants in isotropic turbulence," Physics of Fluids 10, 2336-2346 (1998).

${ }^{67}$ A. Ooi, J. Martin, J. Soria, and M. S. Chong, "A study of the evolution and characteristics of the invariants of the velocity-gradient tensor in isotropic turbulence," Journal of Fluid Mechanics 381, 141-174 (1999).

${ }^{68}$ A. M. Steinberg, B. Coriton, and J. H. Frank, "Influence of combustion on principal strain-rate transport in turbulent premixed flames," Proceedings of the Combustion Institute 35, 1287-1294 (2015). 